\title{
Search for resonant pair production of Higgs bosons decaying to bottom quark-antiquark pairs in proton-proton collisions at $13 \mathrm{TeV}$
}

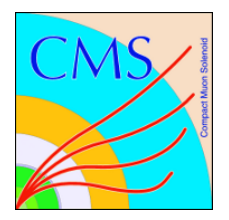

\section{The CMS collaboration}

E-mail: cms-publication-committee-chair@cern.ch

ABSTRACT: A search for a narrow-width resonance decaying into two Higgs bosons, each decaying into a bottom quark-antiquark pair, is presented. The search is performed using proton-proton collision data corresponding to an integrated luminosity of $35.9 \mathrm{fb}^{-1}$ at $\sqrt{s}=13 \mathrm{TeV}$ recorded by the CMS detector at the LHC. No evidence for such a signal is observed. Upper limits are set on the product of the production cross section for the resonance and the branching fraction for the selected decay mode in the resonance mass range from 260 to $1200 \mathrm{GeV}$.

Keywords: Beyond Standard Model, Hadron-Hadron scattering (experiments), Higgs physics

ARXIV EPRINT: 1806.03548 


\section{Contents}

1 Introduction 1

2 Detector and simulated samples $\quad 2$

3 Event reconstruction 3

4 Event selection $\quad 3$

5 Signal and background modeling 5

6 Systematic uncertainties $\quad 8$

$\begin{array}{lll}7 & \text { Results } & 10\end{array}$

$\begin{array}{lll}8 & \text { Summary } & 14\end{array}$

$\begin{array}{ll}\text { The CMS collaboration } & 19\end{array}$

\section{Introduction}

The discovery of a Higgs boson $(\mathrm{H})[1-3]$, with mass of $125 \mathrm{GeV}[4,5]$ and properties consistent with the standard model (SM) of particle physics at the CERN LHC, motivates searches for resonances via their decays into Higgs bosons. Several theories for physics beyond the SM posit narrow-width resonances decaying into pairs of Higgs bosons (HH). For instance, models with a warped extra dimension [6] predict the existence of new particles such as the spin-0 radion [7-9] and the spin-2 first Kaluza-Klein (KK) excitation of the graviton [10-12], which could decay to HH. These models have an extra warped spatial dimension compactified between two branes, with an exponential metric $\kappa l, \kappa$ being the curvature and $l$ the coordinate of the extra spatial dimension [13]. The benchmark of the model is the ultraviolet cutoff of the theory, $\Lambda \equiv \sqrt{8 \pi} e^{-\kappa l} M_{\mathrm{Pl}}, M_{\mathrm{Pl}}$ being the Planck scale. In proton-proton (pp) collisions at the LHC, the graviton and the radion are produced primarily through gluon-gluon fusion and are predicted to decay to $\mathrm{HH}$ with branching fractions of approximately 10 and $23 \%$, respectively [14].

Previous searches for resonant $\mathrm{HH}$ production have been performed by the ATLAS and CMS Collaborations with pp collisions at $\sqrt{s}=8$ and $13 \mathrm{TeV}$. The decay channels studied include $\mathrm{b} \overline{\mathrm{b}} \mathrm{b} \overline{\mathrm{b}}$ [15-17], $\mathrm{b} \overline{\mathrm{b}} \tau \tau$ [18], $\mathrm{b} \overline{\mathrm{b}} \gamma \gamma[19,20], \gamma \gamma \mathrm{WW}$ [21], and $\mathrm{b} \overline{\mathrm{b}} \mathrm{WW}$ [22].

This paper reports the results of a search for narrow-width resonances in the 260-1200 GeV mass range, decaying into a pair of Higgs bosons, each decaying into a pair of bottom quarks. The search is performed using pp collision data collected at $\sqrt{s}=13 \mathrm{TeV}$ 
with the CMS detector at the CERN LHC, corresponding to an integrated luminosity of $35.9 \mathrm{fb}^{-1}$. The main challenge of this search is to discriminate the final-state signature of four bottom quark jets from the overwhelming multijet quantum chromodynamics (QCD) background. This is addressed by dedicated online selection criteria that include b jet identification and by a model of the multijet background that is tested in control regions of data. The analysis closely follows the approach adopted for the $8 \mathrm{TeV}$ data [15] but the sensitivity for high resonance mass values is enhanced because of the significant increase in production cross section at $13 \mathrm{TeV}$, a new trigger strategy and a more efficient algorithm for identifying jets originating from bottom quarks.

\section{Detector and simulated samples}

The central feature of the CMS apparatus is a superconducting solenoid of $6 \mathrm{~m}$ internal diameter, providing a magnetic field of $3.8 \mathrm{~T}$. A silicon pixel and strip tracker, a lead tungstate crystal electromagnetic calorimeter, and a brass and scintillator hadron calorimeter, each composed of a barrel and two endcap sections, reside within the solenoid. Forward calorimeters extend the pseudorapidity $(\eta)$ [23] coverage provided by the barrel and endcap detectors. Muons are detected in gas-ionization chambers embedded in the steel flux-return yoke outside the solenoid. A more detailed description of the CMS detector, together with a definition of the coordinate system used and the relevant kinematic variables, can be found in ref. [23]

Simulated samples of signal events are produced using various Monte Carlo (MC) event generators, with the CMS detector response modeled using the GEANT4 [24] program. To model the production of a generic narrow-width spin-0 resonance, we use an MC simulation of the bulk radion produced through gluon fusion. The momenta and angular distributions for decay products of a spin-2 resonance are distinct from those for a spin-0 resonance, and result in different kinematic distributions. Therefore, we evaluate the signal efficiencies for a narrow-width spin-2 resonance from a separate simulation of the first excitation of a bulk KK graviton produced through gluon fusion and forced to decay to a pair of Higgs bosons with the parameters reported in ref. [25]. Bulk graviton and radion signal events are simulated with masses in the range $260-1200 \mathrm{GeV}$ and widths of $1 \mathrm{MeV}$ (narrow-width approximation), using the MADGRAPH5_aMC@NLO 2.3.3 [26] event generator at leading order (LO). The resonance is forced to decay into a pair of Higgs bosons which in turn decay into $b \bar{b}$. The parton distribution function (PDF) set NNPDF3.0 [27] with LO accuracy is used. The showering and hadronization of partons are simulated with PYTHIA 8.212 [28].

During the 2016 data-taking period the average number of pp interactions per bunch crossing was approximately 23. The simulated samples include these additional pp interactions, referred to as pileup interactions (or pileup), that overlap with the event of interest in the same bunch crossing. Simulated events are weighted to match the number of pp interactions per event in data. 


\section{Event reconstruction}

The particle-flow (PF) algorithm [29] is used to reconstruct and identify individual particle in an event with an optimized combination of information from the various elements of the CMS detector. The algorithm identifies each reconstructed particle (PF candidate) as an electron, a muon, a photon, or a charged or neutral hadron.

The reconstructed vertex with the largest value of summed physics-object transverse momentum squared $\left(p_{T}^{2}\right)$ is taken to be the primary pp interaction vertex. The physics objects are the jets, clustered using the jet finding algorithm [30, 31] with the tracks assigned to the vertex as inputs, and the associated missing transverse momentum, taken as the negative vector sum of the $p_{\mathrm{T}}$ of those jets. This vertex is used for all the objects in the event reconstructed with the $\mathrm{PF}$ algorithm.

Jets are reconstructed from PF candidates using the anti- $k_{\mathrm{T}}$ clustering algorithm [30], with a distance parameter of 0.4 , as implemented in the FASTJET package [31, 32]. Jet identification criteria are also applied to reject jets originating from detector noise. The average neutral energy density from pileup interactions is evaluated from PF objects and subtracted from the reconstructed jets [33]. Jet energy corrections are derived from the simulation, and are confirmed with in situ measurements of the energy balance in dijet and photon+jet events [34].

Jets are identified as originating from b quarks ("b jets") using the DeEPCSV [35] discriminator, a new b tagging algorithm based on a deep neural network with four hidden layers [36]. The DEEPCSV discriminator employs the same set of observables as those used by the combined secondary vertex (CSV) algorithm [35, 37], except that the track selection is expanded to include up to six tracks, further improving the $\mathrm{b}$ jet identification. The operating point chosen corresponds to a $1(12) \%$ rate for misidentifying a light-flavor (c-flavor) jet as a b jet. The $\mathrm{b}$ tagging efficiency for jets with $p_{\mathrm{T}}$ in the $30-150 \mathrm{GeV}$ range is approximately $69 \%$ and gradually decreases for lower and higher jet $p_{\mathrm{T}}$ [35].

\section{Event selection}

The search for a narrow-width $\mathrm{X} \rightarrow \mathrm{H}(\mathrm{b} \overline{\mathrm{b}}) \mathrm{H}(\mathrm{b} \overline{\mathrm{b}})$ resonance is performed for mass values in the $260-1200 \mathrm{GeV}$ range. The angular distributions for the decay products of such a resonance vary substantially over this range. In order to increase the sensitivity of this search, different criteria are used for events in two distinct mass regions: the low-mass region (LMR), for resonance masses from 260 to $620 \mathrm{GeV}$, and the medium-mass region (MMR), for masses from 550 to $1200 \mathrm{GeV}$. The boundary between the LMR and the MMR is at $580 \mathrm{GeV}$. It has been chosen by optimizing for the expected sensitivity and takes into account the uncertainties associated with the background modeling. The mass range above $1200 \mathrm{GeV}$ (high-mass region) is not covered by this search. Above $900 \mathrm{GeV}$, the Higgs bosons have a momentum considerably higher than their mass and the Higgs to b $\bar{b}$ decays are reconstructed more efficiently as single hadronic jets with a larger anti- $k_{\mathrm{T}}$ distance parameter (0.8) [38]. 
Events are selected online by combining two different trigger selections to identify b jets, both using the CSV algorithm. For the first trigger selection, four jets with $p_{\mathrm{T}}>$ $30 \mathrm{GeV}$ and $|\eta|<2.4$ are required. The latter requirement ensures that the jet lies within the tracker acceptance. Of those four jets, two are required to have $p_{\mathrm{T}}>90 \mathrm{GeV}$ and at least three jets are required to be tagged as $\mathrm{b}$ jets. The second trigger selection requires four jets with $p_{\mathrm{T}}>45 \mathrm{GeV}$ and at least three of those jets identified as b jets.

Events are selected offline by requiring at least four b tagged jets with $p_{\mathrm{T}}>30 \mathrm{GeV}$ and $|\eta|<2.4$. The selected jets are combined randomly into pairs to form two Higgs boson candidates with masses $m_{\mathrm{H}_{1}}$ and $m_{\mathrm{H}_{2}}$. For the LMR, HH candidates are chosen from the four selected jets such that $\left|m_{\mathrm{H}}-120 \mathrm{GeV}\right|<40 \mathrm{GeV}$ for each candidate Higgs boson. For the MMR the $\mathrm{H}$ candidates are selected using $\Delta R=\sqrt{(\Delta \eta)^{2}+(\Delta \phi)^{2}}$ less than 1.5, where $\Delta \eta$ and $\Delta \phi$ are the differences in the pseudorapidities and azimuthal angles (in radians) of the two jets.

In the two-dimensional space defined by the reconstructed masses of the two Higgs boson candidates, $\mathrm{H}_{1}$ and $\mathrm{H}_{2}$, a circular signal region (SR) is defined with $\mathcal{R}<1$, where $\mathcal{R}$ is defined as:

$$
\mathcal{R}=\sqrt{\left(\frac{m_{\mathrm{H}_{1}}-\mathcal{M}}{r}\right)^{2}+\left(\frac{m_{\mathrm{H}_{2}}-\mathcal{M}}{r}\right)^{2}}
$$

The central mass value $(\mathcal{M})$ is the average of the means of the $m_{\mathrm{H}_{1}}$ and $m_{\mathrm{H}_{2}}$ distributions for simulated signal events and the parameter $r$ is set to $20 \mathrm{GeV}$. The centers of these circular regions have been determined separately for the LMR and MMR and found to be 120 and $125 \mathrm{GeV}$, respectively. If there are multiple $\mathrm{HH}$ candidates in an event, the combination that minimizes $\mathcal{R}^{2}$ is used.

After these event selection criteria are applied, the dijet invariant mass resolution for $m_{\mathrm{H}_{1}}$ and $m_{\mathrm{H}_{2}}$ is approximately $10-13 \%$, depending on the $p_{\mathrm{T}}$ of the reconstructed Higgs boson, with a few percent shift in the value of the mass peak, relative to $125 \mathrm{GeV}$. The Higgs boson mass resolution is further improved by applying multivariate regression techniques similar to those used in the searches for SM Higgs bosons decaying to $b \bar{b}$ in CMS [39, 40]. The regression estimates a correction that is applied after the standard CMS jet energy corrections [34, 41], and it is computed for individual b jets to improve the accuracy of the measured energy with respect to the b quark energy. To this end, a specialized boosted decision tree [42] is trained on simulated b jets from t $\bar{t}$ events, with inputs that include observables related to the jet structure and b tagging information. The average improvement in the Higgs boson mass resolution, measured with simulated signal samples, is $6-12 \%$, depending on the $p_{\mathrm{T}}$ of the reconstructed Higgs boson. The use of the regression technique increases the sensitivity of the analysis by $5-20 \%$ depending on the mass hypothesis. The regression technique is validated with data samples of $\mathrm{Z} \rightarrow(\mathrm{ee}, \mu \mu)$

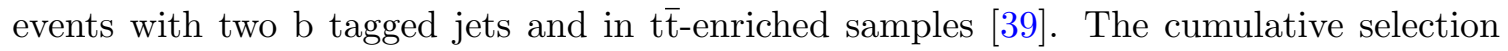
efficiencies of the selection criteria described above for the graviton and radion signal benchmarks are reported in figure 1.

The reconstructed resonance mass $\left(m_{\mathrm{X}}\right)$, computed as the invariant mass of $\mathrm{H}_{1}$ and $\mathrm{H}_{2}$, is displayed for simulated signal events with different mass hypotheses in figure 2 . In 

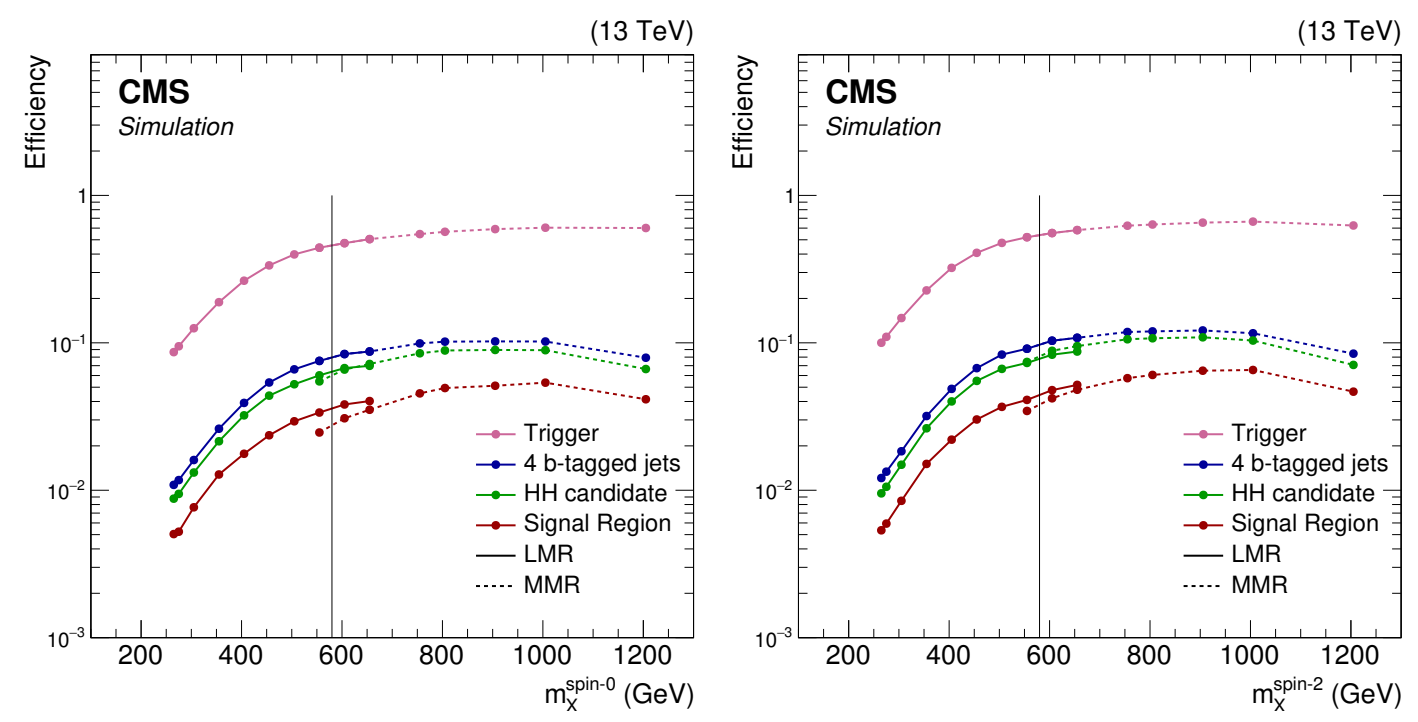

Figure 1. The selection efficiency for simulated $X \rightarrow H(b \bar{b}) H(b \bar{b})$ events for a spin-0 radion (left) and a spin-2 bulk KK-graviton (right), at different stages of the event selection for each mass hypothesis, for the low-mass region (solid) and the medium-mass region (dashed). The vertical line at $580 \mathrm{GeV}$ corresponds to the transition between the LMR and MMR regions.

order to improve the resolution for the resonance mass, the momenta of the reconstructed b quarks are corrected by constraining the invariant mass of the Higgs boson candidates to be $125 \mathrm{GeV}$. Since jet direction is reconstructed with better resolution than jet $p_{\mathrm{T}}$, this constraint mainly benefits the latter. The improvement in resolution for the reconstructed signal resonance ranges from 20 to $40 \%$ depending on the mass hypothesis, resulting in an improvement of the sensitivity by $10-20 \%$. The shift in the reconstructed $m_{\mathrm{X}}$, due to these corrections is also shown in figure 2 . The mass shift is linear in $m_{\mathrm{X}}$ and is caused by the asymmetry of the corrections due to the jet momentum resolution across the $p_{\mathrm{T}}$ range considered.

\section{$5 \quad$ Signal and background modeling}

To search for signal events with various mass hypotheses we fit the $m_{\mathrm{X}}$ distribution for data events in the SR to the sum of two parametric models, one for the signal and one for the SM background, which is dominated by multijet production. This procedure is performed independently in three regions: two within the LMR, as described below, and the MMR. Quantum interference between the signal and non-resonant SM background processes is neglected.

The parametric signal models are built by fitting the $m_{\mathrm{X}}$ distributions obtained from simulated signal events. The shape of the signal $m_{\mathrm{X}}$ distribution is different for the LMR and the MMR, as a result of the different event selection criteria. In the LMR, a sum of two Gaussian functions is used, to account for the effect of reconstructing the two Higgs boson candidates using jets not originating from their decays. This occurs in about $30 \%$ of the events for the lowest resonance mass hypothesis, decreasing to about $5 \%$ at the highest resonance mass hypothesis. Five parameters are required, the mean and width of the two 


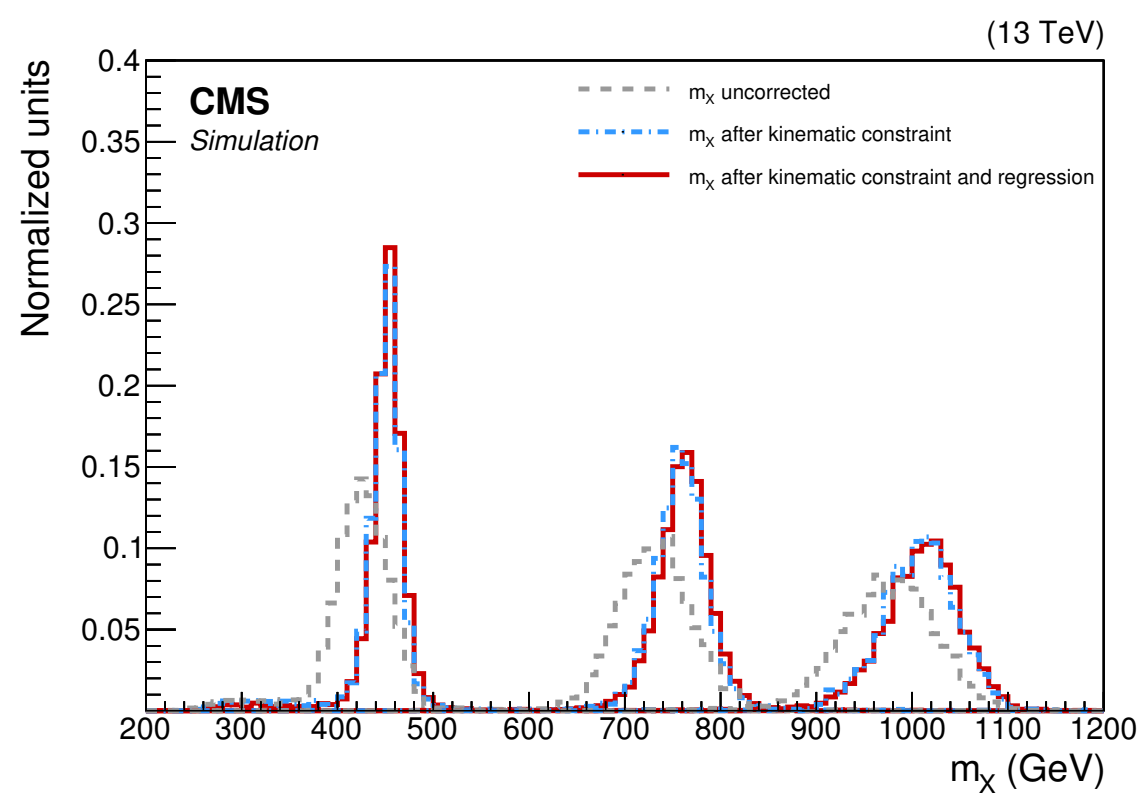

Figure 2. The $m_{\mathrm{X}}$ distribution for simulated signal events (spin-2 bulk KK-graviton) after the event selection criteria for the 450,750 , and $1000 \mathrm{GeV}$ mass hypotheses, with and without the correction obtained by constraining $m_{\mathrm{H}}$ (kinematic constraint) and the specific b jet energy corrections (regression). The distributions are normalized so that the area under the curve for each mass is the same.

Gaussian functions and the ratio of their integrals. In the MMR, the signal is modeled with a function that has a Gaussian core smoothly extended on both sides via exponential tails. This requires two parameters for the mean and width of the Gaussian function, and two parameters for the exponential tails [15].

The parametric function used to model the background $m_{\mathrm{X}}$ distribution is obtained from control regions in data in which events are expected to have kinematic properties similar to events in the SR. These control regions are: (i) sideband regions (SB) defined as $1<\mathcal{R}<2$ and $\left(m_{\mathrm{H}_{1}}-\mathcal{M}\right)\left(m_{\mathrm{H}_{2}}-\mathcal{M}\right)<0$ in the $m_{\mathrm{H}_{1}}-m_{\mathrm{H}_{2}}$ plane (as shown in figure 3), and (ii) events in the SR and both SB regions that do not qualify as signal events due to a requirement that one of the four jets used to reconstruct $m_{\mathrm{X}}$ is not identified as a $\mathrm{b}$ jet (anti-tag selection). For the LMR selection, the $m_{\mathrm{X}}$ distributions in all control regions exhibit a kinematic turn-on, due to the trigger requirements, followed by a smoothly falling distribution at the larger $m_{\mathrm{X}}$ values. The $m_{\mathrm{X}}$ distributions in all control regions for the MMR selection exhibit a similar smoothly falling shape with increasing $m_{\mathrm{X}}$. This feature allows the adoption of a common form of the parametric background model in the SR and control regions. The parameters of the background model are determined by the fit used to extract a possible signal. In the following we describe the derivation of the background model from the different control regions. For the LMR, it is difficult to model simultaneously the turn-on and the tail of the $m_{\mathrm{X}}$ distribution. Thus, the modeling of the background is split into two ranges, LMR1 and LMR2, with two different parametric models to accommodate either a turn-on shape or a falling distribution, respectively. 


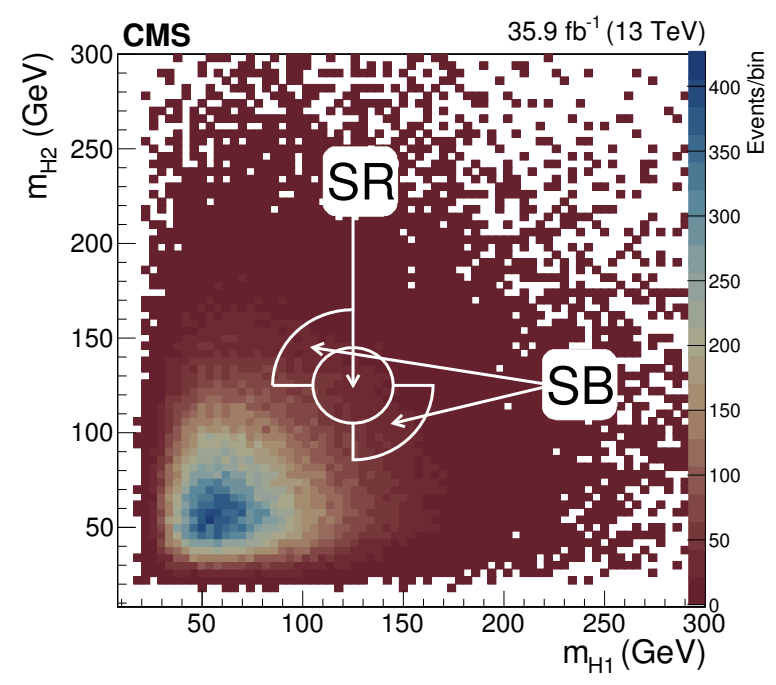

Figure 3. Definition of the SR and the SB in the $\left(m_{\mathrm{H}_{1}}, m_{\mathrm{H}_{2}}\right)$ plane used to motivate and validate the parametric model for the multijet background. The quantities $m_{\mathrm{H}_{1}}$ and $m_{\mathrm{H}_{2}}$ are the two reconstructed Higgs boson masses after applying the multivariate regression described in section 4 . Data corresponds to a selection in the MMR.

An indirect method of determining the boundary between LMR1 and LMR2 is used to avoid revealing the $m_{\mathrm{X}}$ distribution in the SR before fitting for a possible signal. The method uses two selections in the SR and SB, as described in table 1. For the background, the shape of the $m_{\mathrm{X}}$ distribution in the SR (A) is predicted from the shape of the $m_{\mathrm{X}}$ distribution in the SR using the anti-tag selection (C) but reshaped by the ratio, as a function of $m_{\mathrm{X}}$, between the $m_{\mathrm{X}}$ distribution in the SB with the nominal selection (B) and the $m_{\mathrm{X}}$ distribution in the $\mathrm{SB}$ with the anti-tag selection (D), so that $\mathrm{A}^{\prime}=\mathrm{CB} / \mathrm{D}$. To test the validity of this method, new SR and $\mathrm{SB}$ regions, centered at $150 \mathrm{GeV}$, are defined along the diagonal in figure 3 in a similar fashion to those centered at $120 \mathrm{GeV}$. In that case the prediction $\mathrm{E}^{\prime}=\mathrm{GF} / \mathrm{H}$ for the $m_{\mathrm{X}}$ distribution in the $\mathrm{SR}$ region centered at $150 \mathrm{GeV}$ can be tested by directly comparing to the $m_{\mathrm{X}}$ distribution in that region. Figure 4 shows the agreement between this prediction and the observed distribution, validating the use of this method for the SR distribution centered at $120 \mathrm{GeV}$. The boundary between LMR1 and LMR2 is then set at $m_{\mathrm{X}}=310 \mathrm{GeV}$, by optimizing for the expected sensitivity.

The signal and background are evaluated independently in LMR $1=[260,310] \mathrm{GeV}$, $\mathrm{LMR} 2=[310,580] \mathrm{GeV}$ and the $\mathrm{MMR}=[580,1200] \mathrm{GeV}$. For LMR1, a function with a Gaussian core smoothly extended to an exponential tail on the high side is fitted to the $m_{\mathrm{X}}$ distribution [15], while the function defined in ref. [43] eq. (9) is used in LMR2 and the MMR. This function was originally used to describe a Compton spectrum and has three free parameters describing the mean, width and extent of the right side tail. In each case, the goodness of the fit, characterized by the $\chi^{2}$ per degree of freedom, is found to be reasonable. The fit of the background model on the SB for the MMR is shown in figure 5 .

In the absence of a theoretical prediction for the background model, an alternative one based on the Crystal Ball function [44] has been studied, and the difference between 


\begin{tabular}{|lcccc|}
\hline & \multicolumn{2}{c|}{$\mathcal{M}=120 \mathrm{GeV}$} & \multicolumn{2}{c|}{$\mathcal{M}=150 \mathrm{GeV}$} \\
\hline & $\mathrm{SR}$ & $\mathrm{SB}$ & $\mathrm{SR}$ & $\mathrm{SB}$ \\
4 b tagged jets & $\mathrm{A}$ & $\mathrm{B}$ & $\mathrm{E}$ & $\mathrm{F}$ \\
anti-tag & $\mathrm{C}$ & $\mathrm{D}$ & $\mathrm{G}$ & $\mathrm{H}$ \\
\hline
\end{tabular}

Table 1. Definitions of the control regions we used to test the functional form as described in the text.

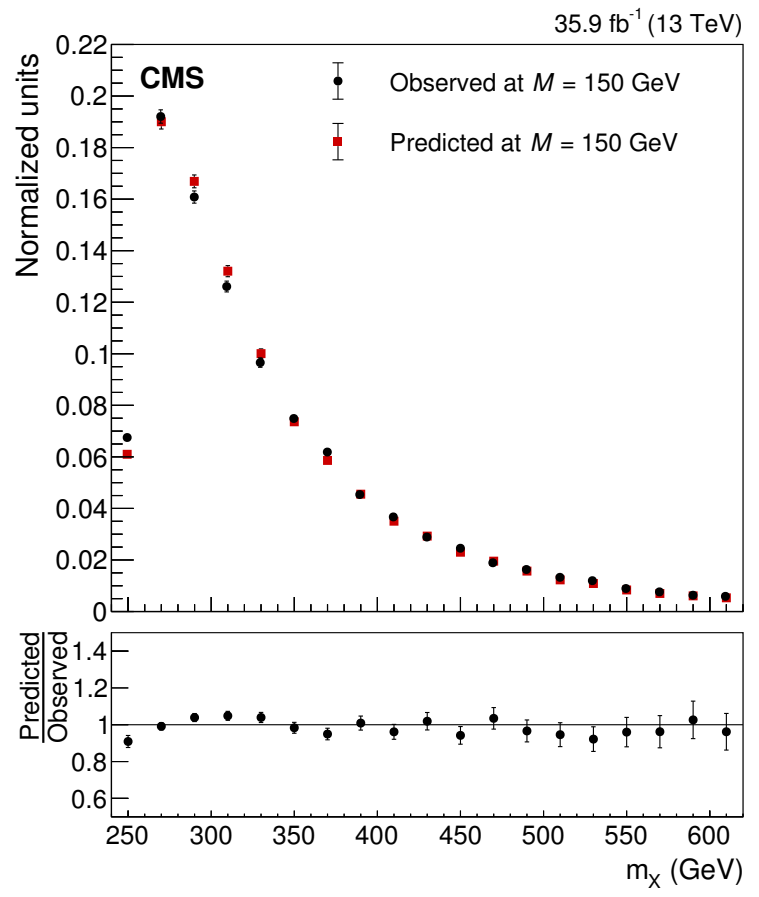

Figure 4. The predicted $m_{\mathrm{X}}$ distribution in data for the LMR (squares) and the observed distribution in a SR (circles) centered at a Higgs boson mass of $150 \mathrm{GeV}$.

them drives the assessment of a systematic uncertainty due to the choice of the particular model. Pseudo-datasets are generated from the alternative function and fitted with the nominal functions to compute biases in the reconstructed signal strength. This procedure is performed for each mass hypothesis and the corresponding biases range between $30-80 \mathrm{fb}$ for the LMR, and $0.1-4 \mathrm{fb}$ for the MMR.

The SM top quark pair production in the SR is estimated from simulation to contribute up to 10 and $15 \%$ of the selected events in the LMR and the MMR, respectively. Since the $t \bar{t}$ contribution to the background exhibits a shape very similar to that for the multijet process, it is implicitly included in the data driven estimate.

\section{$6 \quad$ Systematic uncertainties}

Sources of systematic uncertainties that affect the signal yields are listed in table 2 . The signal yield for a given production cross section is affected by a $2.5 \%$ systematic uncertainty in the measurement of the integrated luminosity at CMS [45]. The uncertainty in the signal 


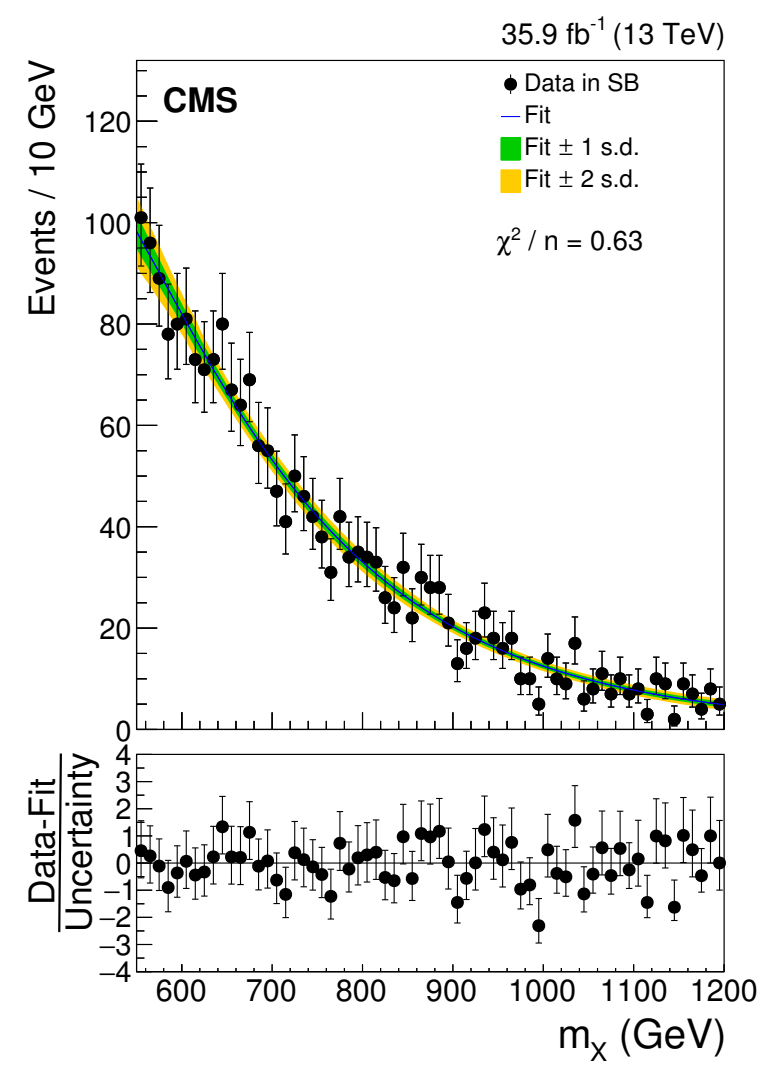

Figure 5. The $m_{X}$ distribution in the SB of the MMR and the fit to the background multijet shape are shown. The shaded regions correspond to variations of \pm 1 and \pm 2 standard deviation (s.d.) in this parametrized form. Here $n$ is the number of degrees of freedom in each fit. The lower panel shows the difference between the data and the fits, divided by the uncertainty in the number of data events.

normalization caused by the choice of the PDF set [46] contributes up to $3.5 \%$. The jet energy scale $[34,41]$ is varied within one standard deviation as a function of jet $p_{\mathrm{T}}$ and $\eta$, and the efficiency of the selection criteria recomputed. The signal efficiencies are found to vary by up to $2.9 \%$. The effect of the uncertainty in the jet energy resolution $[34,41]$ is evaluated by smearing the jet energies according to the measured uncertainty. This causes variations in the signal efficiency of between 0.9 and $2.1 \%$. The trigger efficiency is evaluated in a t $\overline{\mathrm{t}}$-enriched control sample in which an isolated muon is required in addition to the four $\mathrm{b}$ tagged jets with $p_{\mathrm{T}}>30 \mathrm{GeV}$ and $|\eta|<2.4$ demanded in the analysis. The efficiency of each online kinematic and b tagging requirement is evaluated separately and then the efficiency of all the trigger selection criteria is computed in terms of conditional probability. The associated systematic uncertainties are found to impact signal efficiencies at the level of 5-9\%. Signal yields are corrected to match the b tagging efficiency measured in data [35]. The associated uncertainty is evaluated to be about $6-8 \%$.

The systematic uncertainty associated with the choice of the parametric background model is evaluated as described in section 5. We account for the bias as a signal-shaped systematic uncertainty in the background model with normalization centered at zero and 


\begin{tabular}{|lcc|}
\hline Source of & Impact in LMR (\%) & Impact in MMR (\%) \\
systematic uncertainty & Signal & Signal \\
\hline Luminosity & 2.5 & 2.5 \\
Jet energy scale & $0.2-1.8$ & $0.9-2.9$ \\
Jet energy resolution & $0.9-2.1$ & $1.0-1.5$ \\
b tagging scale factor & $6.5-6.9$ & $6.9-8.6$ \\
Trigger efficiency & $6.4-9.0$ & $5.3-7.0$ \\
PDF & $1.5-2.2$ & $2.1-3.5$ \\
\hline
\end{tabular}

Table 2. Impact of systematic uncertainties on the signal efficiencies in the LMR and the MMR.

a Gaussian uncertainty with standard deviation equal to the bias. The impact on the expected limit ranges between $0.3-1.5 \%$.

\section{Results}

The $m_{\mathrm{X}}$ distribution for data in the SR and results of the fit to the parametric background model are shown in figures 6, for the LMR1 and the LMR2, and 7 for the MMR. Parameters controlling the shapes and yields of the signal are allowed to float within ranges determined by the systematic uncertainties. The parameters and normalization of the multijet background shape are left to float freely in the fit. The shapes of the background-only fit are found to adequately describe the data in each of the search region.

The observed and expected upper limits on the cross section times branching fraction for $\mathrm{pp} \rightarrow \mathrm{X} \rightarrow \mathrm{H}(\mathrm{b} \overline{\mathrm{b}}) \mathrm{H}(\mathrm{b} \overline{\mathrm{b}})$ at $95 \%$ confidence level (CL) are computed using the modified frequentist $\mathrm{CL}_{\mathrm{s}}$ method [47-50]. These limits are shown in figures 8 and 9 for spin-2 and spin-0 hypotheses, respectively. The green and yellow bands represent the \pm 1 and \pm 2 standard deviation confidence intervals around the expected limits. The observed limits in these figures exhibit several deviations from the expected one beyond \pm 2 standard deviation. The local $p$-value of the most significant excess (deficit) is $2.6(-3.6)$ standard deviation. In order to estimate the global significance of these deviations within the search range, we estimated, based on pseudo-experiments, a global probability to see three (four) positive or negative deviations from the expected limits consistent with narrow positive or negative signal contributions with the square sum of the significances exceeding 5.1 (5.6) standard deviation, as observed in data. In both cases, the global probability was found to be in a percent range, consistent with a statistical nature of the observed deviations. In figure 8, the NLO theoretical cross section for the gluon fusion production of a bulk KKgraviton with $\kappa l$ and $\kappa$ set to 35 and $0.5 M_{\mathrm{Pl}}$ respectively is shown. This KK-graviton is excluded at $95 \% \mathrm{CL}$ in the mass ranges of $320-450$ and $480-720 \mathrm{GeV}$. In figure 9 , the NLO theoretical cross section for the gluon fusion production of a radion with decay constant $\Lambda=3 \mathrm{TeV}$ is shown. Such a radion is excluded at 95\% CL in the mass ranges of 260-280, 300-450, and 480-1120 GeV. 

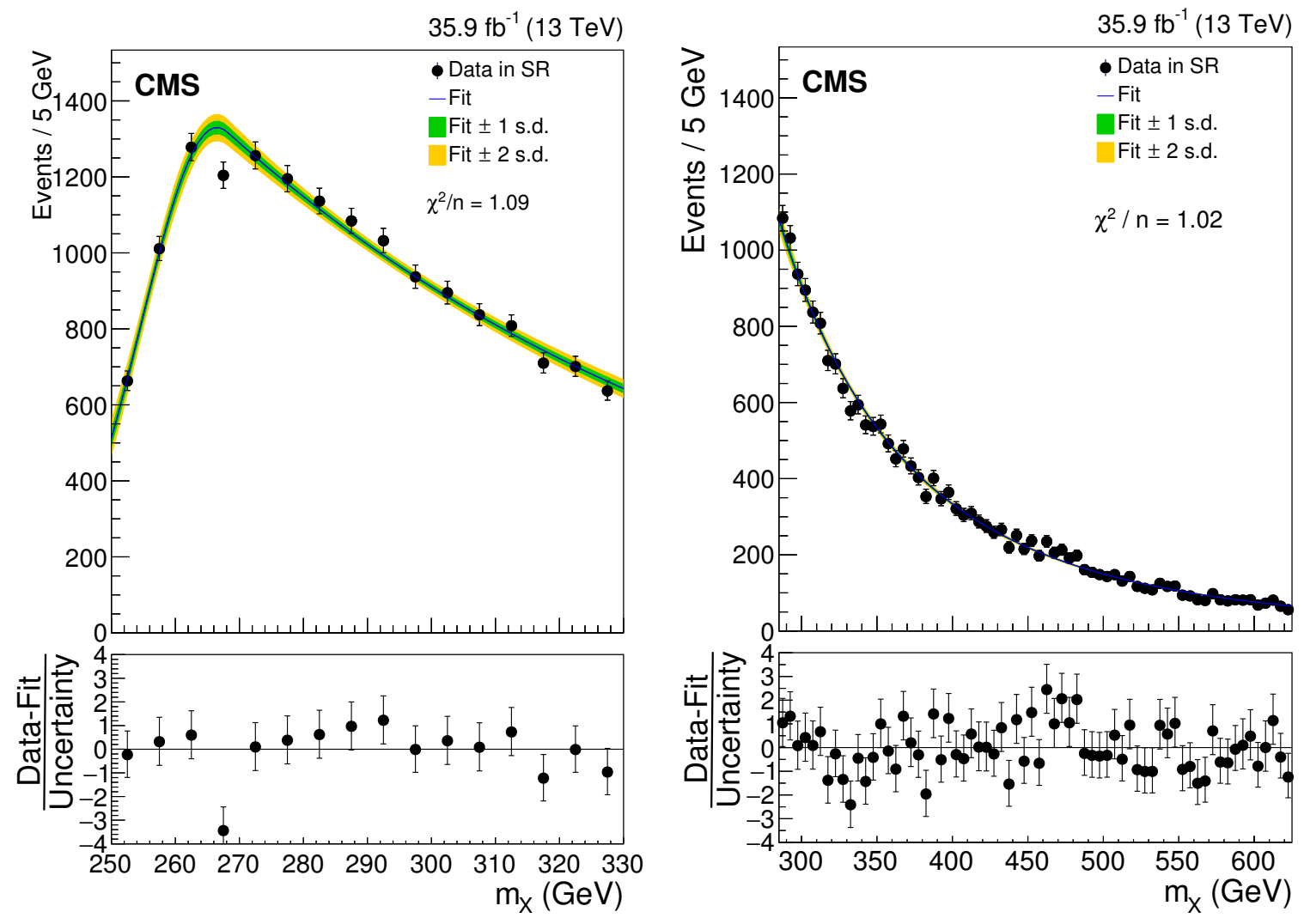

Figure 6. The $m_{\mathrm{X}}$ distributions for LMR1 (left) and LMR2 (right) in the SR. These distributions are fitted in the two ranges to the reference model. A fit to the background-only hypothesis, multijets, is shown. The shaded regions correspond to variations of \pm 1 and \pm 2 standard deviation (s.d.) in the parametrized form. Here $n$ is the number of degrees of freedom in each fit. The lower panels show the difference between the data and the fits, divided by the uncertainty in the number of data events. 


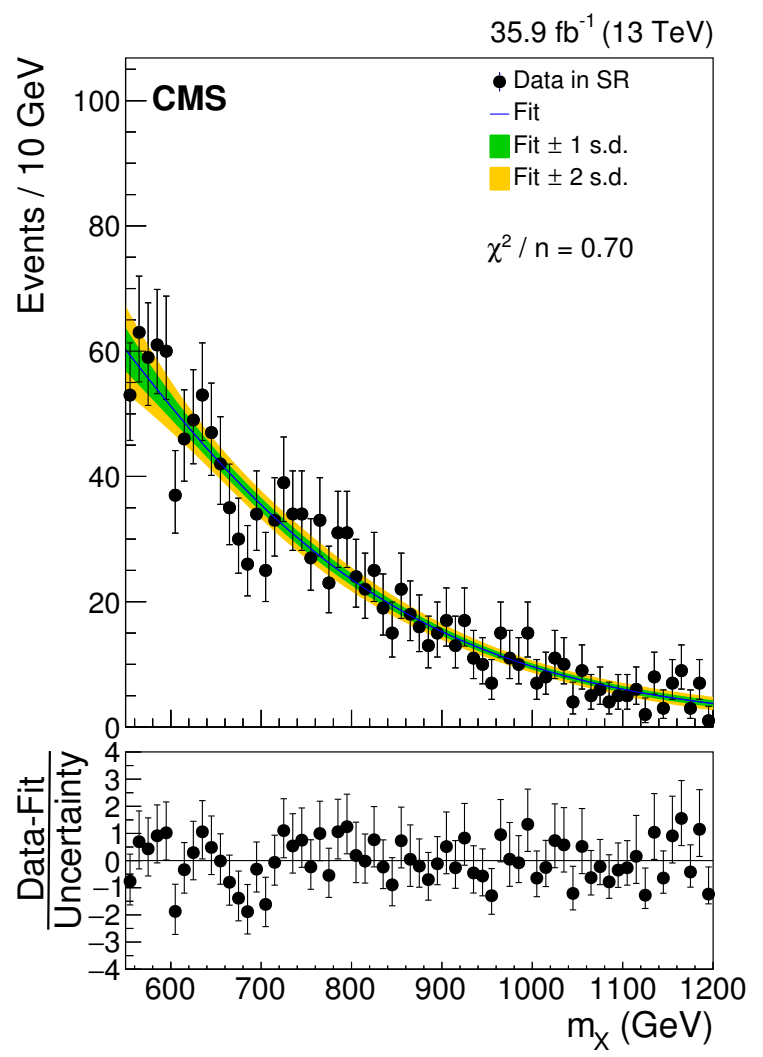

Figure 7. The $m_{\mathrm{X}}$ distribution for the multijet background in the SR in data for the MMR. A fit to the background-only hypothesis, which consists of the multijet shape, is shown. The shaded regions correspond to variations of \pm 1 and \pm 2 standard deviation (s.d.) in this parametrized form. Here $n$ is the number of degrees of freedom in each fit. The lower panel shows the difference between the data and the fits, divided by the uncertainty in the number of data events. 


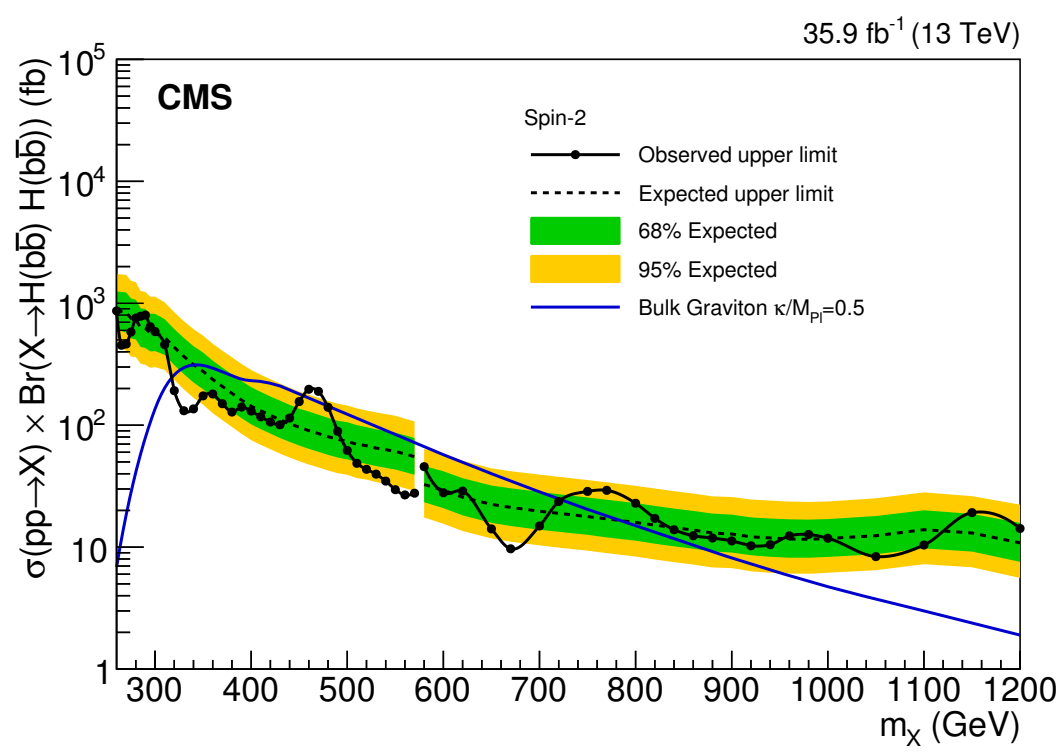

Figure 8. The observed and expected upper limits on the cross section for a spin-2 resonance $\mathrm{X} \rightarrow \mathrm{H}(\mathrm{b} \overline{\mathrm{b}}) \mathrm{H}(\mathrm{b} \overline{\mathrm{b}})$ at $95 \% \mathrm{CL}$, using the asymptotic $\mathrm{CL}_{\mathrm{s}}$ method. The theoretical cross section for the bulk KK-graviton, with $\kappa / M_{\mathrm{Pl}}=0.5$ and $\kappa l=35$, decaying to four b jets via Higgs bosons is overlaid. The transition between the LMR and the MMR is based on the expected sensitivity, resulting in the observed discontinuity.

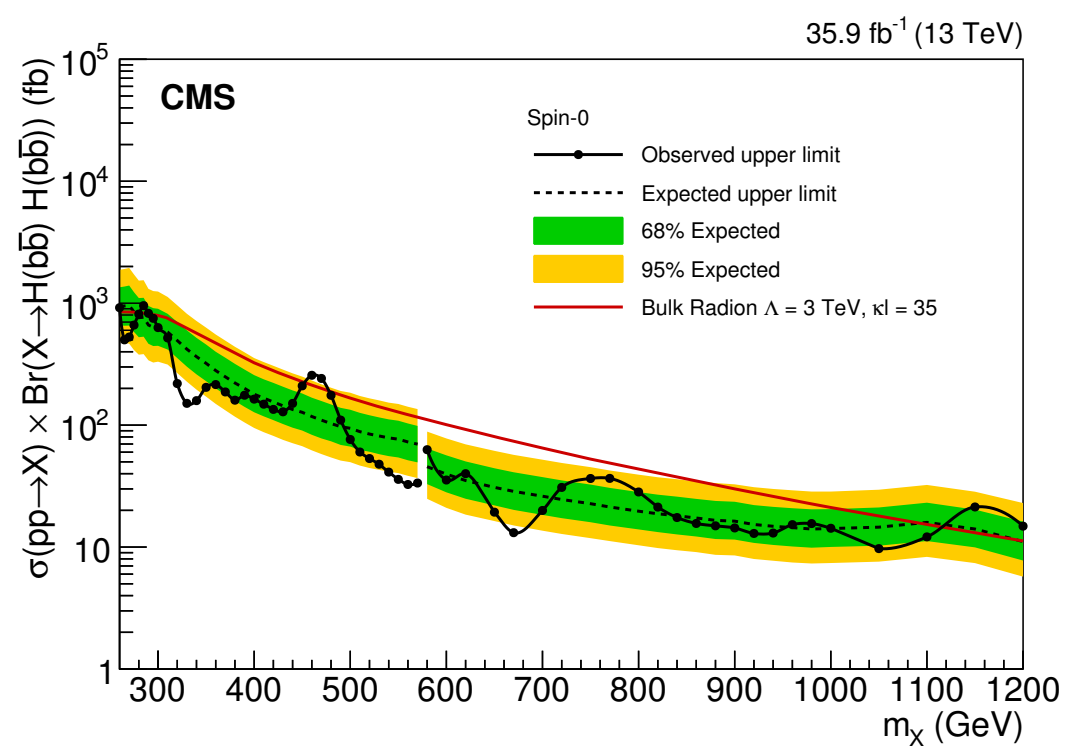

Figure 9. The observed and expected upper limits on the cross section for a spin-0 resonance $\mathrm{X} \rightarrow \mathrm{H}(\mathrm{b} \overline{\mathrm{b}}) \mathrm{H}(\mathrm{b} \overline{\mathrm{b}})$ at $95 \% \mathrm{CL}$, using the asymptotic $\mathrm{CL}_{\mathrm{s}}$ method. The theoretical cross section for the production of a radion, with $\Lambda=3 \mathrm{TeV}, \kappa l=35$, and no radion-Higgs boson mixing, decaying to four $b$ jets via Higgs bosons is overlaid. The transition between the LMR and the MMR is based on the expected sensitivity, resulting in the observed discontinuity. 


\section{Summary}

A search for a narrow-width resonance decaying into two Higgs bosons, each decaying into a bottom quark-antiquark pair, is presented. The search is performed using protonproton collision data corresponding to an integrated luminosity of $35.9 \mathrm{fb}^{-1}$ at $\sqrt{s}=13 \mathrm{TeV}$ recorded by the CMS detector at the LHC. No evidence for a signal is observed and upper limits at $95 \%$ confidence level on the production cross section for spin-0 and spin-2 resonances in the mass range from 260 to $1200 \mathrm{GeV}$ are set. These cross-section limits are translated into an exclusion at $95 \%$ confidence level of a bulk KK-graviton (with $\kappa l=35$ and $\left.\kappa=0.5 \mathrm{M}_{\mathrm{Pl}}\right)$ in the mass ranges of $320-450 \mathrm{GeV}$ and $480-720 \mathrm{GeV}$. The corresponding excluded mass ranges for a radion (with decay constant $\Lambda=3 \mathrm{TeV}$ ) are $260-280 \mathrm{GeV}$, 300-450 GeV and 480-1120 GeV. This analysis outperforms a similar search by CMS using $17.9 \mathrm{fb}^{-1}$ collected at $8 \mathrm{TeV}[15]$ and extends the sensitivity to the gluon fusion production of a radion with decay constant $\Lambda=3 \mathrm{TeV}$ and to bulk graviton with $\kappa$ set to $0.5 M_{\mathrm{Pl}}$.

\section{Acknowledgments}

We congratulate our colleagues in the CERN accelerator departments for the excellent performance of the LHC and thank the technical and administrative staffs at CERN and at other CMS institutes for their contributions to the success of the CMS effort. In addition, we gratefully acknowledge the computing centres and personnel of the Worldwide LHC Computing Grid for delivering so effectively the computing infrastructure essential to our analyses. Finally, we acknowledge the enduring support for the construction and operation of the LHC and the CMS detector provided by the following funding agencies: BMWFW and FWF (Austria); FNRS and FWO (Belgium); CNPq, CAPES, FAPERJ, FAPERGS, and FAPESP (Brazil); MES (Bulgaria); CERN; CAS, MoST, and NSFC (China); COLCIENCIAS (Colombia); MSES and CSF (Croatia); RPF (Cyprus); SENESCYT (Ecuador); MoER, ERC IUT, and ERDF (Estonia); Academy of Finland, MEC, and HIP (Finland); CEA and CNRS/IN2P3 (France); BMBF, DFG, and HGF (Germany); GSRT (Greece); NKFIA (Hungary); DAE and DST (India); IPM (Iran); SFI (Ireland); INFN (Italy); MSIP and NRF (Republic of Korea); LAS (Lithuania); MOE and UM (Malaysia); BUAP, CINVESTAV, CONACYT, LNS, SEP, and UASLP-FAI (Mexico); MBIE (New Zealand); PAEC (Pakistan); MSHE and NSC (Poland); FCT (Portugal); JINR (Dubna); MON, RosAtom, RAS, RFBR, and NRC KI (Russia); MESTD (Serbia); SEIDI, CPAN, PCTI, and FEDER (Spain); Swiss Funding Agencies (Switzerland); MST (Taipei); ThEPCenter, IPST, STAR, and NSTDA (Thailand); TUBITAK and TAEK (Turkey); NASU and SFFR (Ukraine); STFC (United Kingdom); DOE and NSF (U.S.A.).

Individuals have received support from the Marie-Curie programme and the European Research Council and Horizon 2020 Grant, contract No. 675440 (European Union); the Leventis Foundation; the A. P. Sloan Foundation; the Alexander von Humboldt Foundation; the Belgian Federal Science Policy Office; the Fonds pour la Formation à la Recherche dans l'Industrie et dans l'Agriculture (FRIA-Belgium); the Agentschap voor Innovatie door Wetenschap en Technologie (IWT-Belgium); the F.R.S.-FNRS and FWO (Belgium) under 
the "Excellence of Science - EOS" - be.h project n. 30820817; the Ministry of Education, Youth and Sports (MEYS) of the Czech Republic; the Lendület ("Momentum") Programme and the János Bolyai Research Scholarship of the Hungarian Academy of Sciences, the New National Excellence Program ÚNKP, the NKFIA research grants 123842, 123959, 124845, 124850 and 125105 (Hungary); the Council of Science and Industrial Research, India; the HOMING PLUS programme of the Foundation for Polish Science, cofinanced from European Union, Regional Development Fund, the Mobility Plus programme of the Ministry of Science and Higher Education, the National Science Center (Poland), contracts Harmonia 2014/14/M/ST2/00428, Opus 2014/13/B/ST2/02543, 2014/15/B/ST2/03998, and 2015/19/B/ST2/02861, Sonata-bis 2012/07/E/ST2/01406; the National Priorities Research Program by Qatar National Research Fund; the Programa Estatal de Fomento de la Investigación Científica y Técnica de Excelencia María de Maeztu, grant MDM-2015-0509 and the Programa Severo Ochoa del Principado de Asturias; the Thalis and Aristeia programmes cofinanced by EU-ESF and the Greek NSRF; the Rachadapisek Sompot Fund for Postdoctoral Fellowship, Chulalongkorn University and the Chulalongkorn Academic into Its 2nd Century Project Advancement Project (Thailand); the Welch Foundation, contract C-1845; and the Weston Havens Foundation (U.S.A.).

Open Access. This article is distributed under the terms of the Creative Commons Attribution License (CC-BY 4.0), which permits any use, distribution and reproduction in any medium, provided the original author(s) and source are credited.

\section{References}

[1] ATLAS collaboration, Observation of a new particle in the search for the Standard Model Higgs boson with the ATLAS detector at the LHC, Phys. Lett. B 716 (2012) 1 [arXiv: 1207.7214] [INSPIRE].

[2] CMS collaboration, Observation of a new boson at a mass of $125 \mathrm{GeV}$ with the CMS experiment at the LHC, Phys. Lett. B 716 (2012) 30 [arXiv:1207.7235] [INSPIRE].

[3] CMS collaboration, Observation of a new boson with mass near $125 \mathrm{GeV}$ in pp collisions at $\sqrt{s}=7$ and $8 \mathrm{TeV}$, JHEP 06 (2013) 081 [arXiv:1303.4571] [INSPIRE].

[4] ATLAS, CMS collaborations, Combined Measurement of the Higgs Boson Mass in pp Collisions at $\sqrt{s}=7$ and $8 \mathrm{TeV}$ with the ATLAS and CMS Experiments, Phys. Rev. Lett. 114 (2015) 191803 [arXiv: 1503.07589] [INSPIRE].

[5] CMS collaboration, Measurements of properties of the Higgs boson decaying into the four-lepton final state in pp collisions at $\sqrt{s}=13$ TeV, JHEP 11 (2017) 047 [arXiv: 1706.09936] [INSPIRE].

[6] L. Randall and R. Sundrum, A large mass hierarchy from a small extra dimension, Phys. Rev. Lett. 83 (1999) 3370 [hep-ph/9905221] [INSPIRE].

[7] W.D. Goldberger and M.B. Wise, Modulus stabilization with bulk fields, Phys. Rev. Lett. 83 (1999) 4922 [hep-ph/9907447] [INSPIRE].

[8] O. DeWolfe, D.Z. Freedman, S.S. Gubser and A. Karch, Modeling the fifth-dimension with scalars and gravity, Phys. Rev. D 62 (2000) 046008 [hep-th/9909134] [INSPIRE]. 
[9] C. Csáki, M. Graesser, L. Randall and J. Terning, Cosmology of brane models with radion stabilization, Phys. Rev. D 62 (2000) 045015 [hep-ph/9911406] [inSPIRE].

[10] H. Davoudiasl, J.L. Hewett and T.G. Rizzo, Phenomenology of the Randall-Sundrum gauge hierarchy model, Phys. Rev. Lett. 84 (2000) 2080 [hep-ph/9909255] [INSPIRE].

[11] C. Csáki, M.L. Graesser and G.D. Kribs, Radion dynamics and electroweak physics, Phys. Rev. D 63 (2001) 065002 [hep-th/0008151] [INSPIRE].

[12] K. Agashe, H. Davoudiasl, G. Perez and A. Soni, Warped gravitons at the LHC and beyond, Phys. Rev. D 76 (2007) 036006 [hep-ph/0701186] [INSPIRE].

[13] G.F. Giudice, R. Rattazzi and J.D. Wells, Graviscalars from higher dimensional metrics and curvature Higgs mixing, Nucl. Phys. B 595 (2001) 250 [hep-ph/0002178] [INSPIRE].

[14] A. Oliveira, Gravity particles from warped extra dimensions, predictions for LHC, arXiv: 1404.0102 [INSPIRE].

[15] CMS collaboration, Search for resonant pair production of Higgs bosons decaying to two bottom quark-antiquark pairs in proton-proton collisions at 8 TeV, Phys. Lett. B 749 (2015) 560 [arXiv: 1503.04114] [INSPIRE].

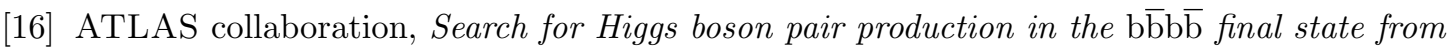
pp collisions at $\sqrt{s}=8 \mathrm{TeV}$ with the ATLAS detector, Eur. Phys. J. C 75 (2015) 412 [arXiv: 1506.00285] [INSPIRE].

[17] ATLAS collaboration, Search for pair production of Higgs bosons in the $\mathrm{b} \overline{\mathrm{b}} \overline{\mathrm{b}}$ final state using proton-proton collisions at $\sqrt{s}=13 \mathrm{TeV}$ with the ATLAS detector, Phys. Rev. D 94 (2016) 052002 [arXiv: 1606. 04782] [INSPIRE].

[18] CMS collaboration, Search for Higgs boson pair production in events with two bottom quarks and two tau leptons in proton-proton collisions at $\sqrt{s}=13 \mathrm{TeV}$, Phys. Lett. B 778 (2018) 101 [arXiv: 1707.02909] [INSPIRE].

[19] CMS collaboration, Search for two Higgs bosons in final states containing two photons and two bottom quarks in proton-proton collisions at 8 TeV, Phys. Rev. D 94 (2016) 052012 [arXiv: 1603.06896] [INSPIRE].

[20] ATLAS collaboration, Search for Higgs boson pair production in the $\gamma \gamma \mathrm{b} \overline{\mathrm{b}}$ final state using pp collision data at $\sqrt{s}=8 \mathrm{TeV}$ from the ATLAS detector, Phys. Rev. Lett. 114 (2015) 081802 [arXiv: 1406 . 5053] [INSPIRE].

[21] ATLAS collaboration, Searches for Higgs boson pair production in the $h h \rightarrow \mathrm{b} \overline{\mathrm{b}} \tau \tau, \gamma \gamma W W^{*}, \gamma \gamma \mathrm{b} \overline{\mathrm{b}}, \mathrm{b} \overline{\mathrm{b}} \mathrm{b} \overline{\mathrm{b}}$ channels with the ATLAS detector, Phys. Rev. D 92 (2015) 092004 [arXiv: 1509.04670] [INSPIRE].

[22] CMS collaboration, Search for resonant and nonresonant Higgs boson pair production in the $\mathrm{b} \overline{\mathrm{b}} l \nu l \nu$ final state in proton-proton collisions at $\sqrt{s}=13 \mathrm{TeV}$, JHEP 01 (2018) 054 [arXiv: 1708.04188] [INSPIRE].

[23] CMS collaboration, The CMS experiment at the CERN LHC, 2008 JINST 3 S08004 [INSPIRE].

[24] GEANT4 collaboration, S. Agostinelli et al., GEANT4 - a simulation toolkit, Nucl. Instrum. Meth. A 506 (2003) 250 [inSPIRE].

[25] M. Gouzevitch, A. Oliveira, J. Rojo, R. Rosenfeld, G.P. Salam and V. Sanz, Scale-invariant resonance tagging in multijet events and new physics in Higgs pair production, JHEP $\mathbf{0 7}$ (2013) 148 [arXiv:1303.6636] [INSPIRE]. 
[26] J. Alwall et al., The automated computation of tree-level and next-to-leading order differential cross sections and their matching to parton shower simulations, JHEP 07 (2014) 079 [arXiv: 1405.0301] [INSPIRE].

[27] NNPDF collaboration, R.D. Ball et al., Parton distributions for the LHC Run II, JHEP 04 (2015) 040 [arXiv: 1410.8849] [INSPIRE].

[28] T. Sjöstrand et al., An introduction to PYTHIA 8.2, Comput. Phys. Commun. 191 (2015) 159 [arXiv: 1410.3012] [INSPIRE].

[29] CMS collaboration, Particle-flow reconstruction and global event description with the CMS detector, 2017 JINST 12 P10003 [arXiv:1706.04965] [INSPIRE].

[30] M. Cacciari, G.P. Salam and G. Soyez, The anti- $k_{t}$ jet clustering algorithm, JHEP 04 (2008) 063 [arXiv: 0802.1189] [INSPIRE].

[31] M. Cacciari, G.P. Salam and G. Soyez, FastJet user manual, Eur. Phys. J. C 72 (2012) 1896 [arXiv:1111.6097] [INSPIRE].

[32] M. Cacciari and G.P. Salam, Dispelling the $N^{3}$ myth for the $k_{t}$ jet-finder, Phys. Lett. B 641 (2006) 57 [hep-ph/0512210] [INSPIRE].

[33] M. Cacciari and G.P. Salam, Pileup subtraction using jet areas, Phys. Lett. B 659 (2008) 119 [arXiv: 0707.1378] [INSPIRE].

[34] CMS collaboration, Jet energy scale and resolution in the CMS experiment in pp collisions at $8 \mathrm{TeV}, 2017$ JINST $12 \mathrm{P} 02014$ [arXiv: 1607.03663] [INSPIRE].

[35] CMS collaboration, Identification of heavy-flavour jets with the CMS detector in pp collisions at $13 \mathrm{TeV}, 2018$ JINST $13 \mathrm{P} 05011$ [arXiv: 1712.07158] [INSPIRE].

[36] D. Guest, J. Collado, P. Baldi, S.-C. Hsu, G. Urban and D. Whiteson, Jet flavor classification in high-energy physics with deep neural networks, Phys. Rev. D 94 (2016) 112002 [arXiv: 1607.08633] [INSPIRE].

[37] CMS collaboration, Identification of b-quark jets with the CMS experiment, 2013 JINST 8 P04013 [arXiv: 1211.4462] [INSPIRE].

[38] CMS collaboration, Search for a massive resonance decaying to a pair of Higgs bosons in the four b quark final state in proton-proton collisions at $\sqrt{s}=13 \mathrm{TeV}$, Phys. Lett. B 781 (2018) 244 [arXiv: 1710.04960$]$ [INSPIRE].

[39] CMS collaboration, Search for the standard model Higgs boson produced in association with a W or a $Z$ boson and decaying to bottom quarks, Phys. Rev. D 89 (2014) 012003 [arXiv: 1310.3687] [INSPIRE].

[40] CMS collaboration, Search for the standard model Higgs boson produced through vector boson

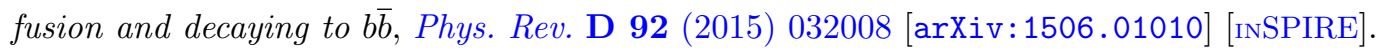

[41] CMS collaboration, Determination of jet energy calibration and transverse momentum resolution in CMS, 2011 JINST 6 P11002 [arXiv:1107.4277] [INSPIRE].

[42] A. Höcker et al., TMVA, the toolkit for multivariate data analysis with ROOT, in XIth International Workshop on Advanced Computing and Analysis Techniques in Physics Research (ACAT), p. 40 (2007) [physics/0703039] [INSPIRE].

[43] BeLLE collaboration, H. Ikeda et al., A detailed test of the CsI(Tl) calorimeter for BELLE with photon beams of energy between 20-MeV and 5.4-GeV, Nucl. Instrum. Meth. A 441 (2000) 401 [INSPIRE].

[44] M.J. Oreglia, A study of the reactions $\psi^{\prime} \rightarrow \gamma \gamma \psi$, Ph.D. Thesis, Stanford University (1980). SLAC Report SLAC-R-236 [INSPIRE]. 
[45] CMS collaboration, CMS luminosity measurements for the 2016 data taking period, CMS-PAS-LUM-17-001 (2017).

[46] J. Butterworth et al., PDF4LHC recommendations for LHC Run II, J. Phys. G 43 (2016) 023001 [arXiv: 1510.03865] [INSPIRE].

[47] G. Cowan, K. Cranmer, E. Gross and O. Vitells, Asymptotic formulae for likelihood-based tests of new physics, Eur. Phys. J. C 71 (2011) 1554 [Erratum ibid. C 73 (2013) 2501] [arXiv: 1007.1727] [INSPIRE].

[48] T. Junk, Confidence level computation for combining searches with small statistics, Nucl. Instrum. Meth. A 434 (1999) 435 [hep-ex/9902006] [INSPIRE].

[49] A.L. Read, Presentation of search results: The $C L_{s}$ technique, J. Phys. G 28 (2002) 2693 [INSPIRE].

[50] ATLAS, CMS collaborations and LHC Higgs Combination Group, Procedure for the LHC Higgs boson search combination in Summer 2011, CMS-NOTE-2011-005 (2011). 


\section{The CMS collaboration}

\section{Yerevan Physics Institute, Yerevan, Armenia}

A.M. Sirunyan, A. Tumasyan

\section{Institut für Hochenergiephysik, Wien, Austria}

W. Adam, F. Ambrogi, E. Asilar, T. Bergauer, J. Brandstetter, E. Brondolin, M. Dragicevic, J. Erö, A. Escalante Del Valle, M. Flechl, M. Friedl, R. Frühwirth ${ }^{1}$, V.M. Ghete, J. Grossmann, J. Hrubec, M. Jeitler ${ }^{1}$, A. König, N. Krammer, I. Krätschmer, D. Liko, T. Madlener, I. Mikulec, E. Pree, N. Rad, H. Rohringer, J. Schieck ${ }^{1}$, R. Schöfbeck, M. Spanring, D. Spitzbart, A. Taurok, W. Waltenberger, J. Wittmann, C.-E. Wulz ${ }^{1}$, M. Zarucki

\section{Institute for Nuclear Problems, Minsk, Belarus}

V. Chekhovsky, V. Mossolov, J. Suarez Gonzalez

\section{Universiteit Antwerpen, Antwerpen, Belgium}

E.A. De Wolf, D. Di Croce, X. Janssen, J. Lauwers, M. Pieters, M. Van De Klundert, H. Van Haevermaet, P. Van Mechelen, N. Van Remortel

\section{Vrije Universiteit Brussel, Brussel, Belgium}

S. Abu Zeid, F. Blekman, J. D'Hondt, I. De Bruyn, J. De Clercq, K. Deroover, G. Flouris, D. Lontkovskyi, S. Lowette, I. Marchesini, S. Moortgat, L. Moreels, Q. Python, K. Skovpen, S. Tavernier, W. Van Doninck, P. Van Mulders, I. Van Parijs

\section{Université Libre de Bruxelles, Bruxelles, Belgium}

D. Beghin, B. Bilin, H. Brun, B. Clerbaux, G. De Lentdecker, H. Delannoy, B. Dorney, G. Fasanella, L. Favart, R. Goldouzian, A. Grebenyuk, A.K. Kalsi, T. Lenzi, J. Luetic, T. Seva, E. Starling, C. Vander Velde, P. Vanlaer, D. Vannerom, R. Yonamine

\section{Ghent University, Ghent, Belgium}

T. Cornelis, D. Dobur, A. Fagot, M. Gul, I. Khvastunov², D. Poyraz, C. Roskas, D. Trocino, M. Tytgat, W. Verbeke, B. Vermassen, M. Vit, N. Zaganidis

\section{Université Catholique de Louvain, Louvain-la-Neuve, Belgium}

H. Bakhshiansohi, O. Bondu, S. Brochet, G. Bruno, C. Caputo, A. Caudron, P. David, S. De Visscher, C. Delaere, M. Delcourt, B. Francois, A. Giammanco, G. Krintiras, V. Lemaitre, A. Magitteri, A. Mertens, M. Musich, K. Piotrzkowski, L. Quertenmont, A. Saggio, M. Vidal Marono, S. Wertz, J. Zobec

\section{Centro Brasileiro de Pesquisas Fisicas, Rio de Janeiro, Brazil}

W.L. Aldá Júnior, F.L. Alves, G.A. Alves, L. Brito, G. Correia Silva, C. Hensel, A. Moraes, M.E. Pol, P. Rebello Teles

Universidade do Estado do Rio de Janeiro, Rio de Janeiro, Brazil

E. Belchior Batista Das Chagas, W. Carvalho, J. Chinellato ${ }^{3}$, E. Coelho, E.M. Da Costa, G.G. Da Silveira ${ }^{4}$, D. De Jesus Damiao, S. Fonseca De Souza, H. Malbouisson, M. Medina Jaime ${ }^{5}$, M. Melo De Almeida, C. Mora Herrera, L. Mundim, H. Nogima, 
L.J. Sanchez Rosas, A. Santoro, A. Sznajder, M. Thiel, E.J. Tonelli Manganote ${ }^{3}$, F. Torres Da Silva De Araujo, A. Vilela Pereira

Universidade Estadual Paulista ${ }^{a}$, Universidade Federal do $\mathrm{ABC}^{b}$, São Paulo, Brazil

S. Ahuja ${ }^{a}$, C.A. Bernardes ${ }^{a}$, L. Calligaris ${ }^{a}$, T.R. Fernandez Perez Tomei ${ }^{a}$, E.M. Gregores $^{b}$, P.G. Mercadante ${ }^{b}$, S.F. Novaes ${ }^{a}$, SandraS. Padula ${ }^{a}$, D. Romero Abad ${ }^{b}$, J.C. Ruiz Vargas ${ }^{a}$

Institute for Nuclear Research and Nuclear Energy, Bulgarian Academy of Sciences, Sofia, Bulgaria

A. Aleksandrov, R. Hadjiiska, P. Iaydjiev, A. Marinov, M. Misheva, M. Rodozov, M. Shopova, G. Sultanov

University of Sofia, Sofia, Bulgaria

A. Dimitrov, L. Litov, B. Pavlov, P. Petkov

Beihang University, Beijing, China

W. Fang ${ }^{6}$, X. Gao ${ }^{6}$, L. Yuan

Institute of High Energy Physics, Beijing, China

M. Ahmad, J.G. Bian, G.M. Chen, H.S. Chen, M. Chen, Y. Chen, C.H. Jiang, D. Leggat, H. Liao, Z. Liu, F. Romeo, S.M. Shaheen, A. Spiezia, J. Tao, C. Wang, Z. Wang, E. Yazgan, H. Zhang, J. Zhao

State Key Laboratory of Nuclear Physics and Technology, Peking University, Beijing, China

Y. Ban, G. Chen, J. Li, Q. Li, S. Liu, Y. Mao, S.J. Qian, D. Wang, Z. Xu

Tsinghua University, Beijing, China

Y. Wang

Universidad de Los Andes, Bogota, Colombia

C. Avila, A. Cabrera, C.A. Carrillo Montoya, L.F. Chaparro Sierra, C. Florez, C.F. González Hernández, M.A. Segura Delgado

University of Split, Faculty of Electrical Engineering, Mechanical Engineering and Naval Architecture, Split, Croatia

B. Courbon, N. Godinovic, D. Lelas, I. Puljak, P.M. Ribeiro Cipriano, T. Sculac

University of Split, Faculty of Science, Split, Croatia

Z. Antunovic, M. Kovac

Institute Rudjer Boskovic, Zagreb, Croatia

V. Brigljevic, D. Ferencek, K. Kadija, B. Mesic, A. Starodumov ${ }^{7}$, T. Susa

University of Cyprus, Nicosia, Cyprus

M.W. Ather, A. Attikis, G. Mavromanolakis, J. Mousa, C. Nicolaou, F. Ptochos, P.A. Razis, H. Rykaczewski

Charles University, Prague, Czech Republic

M. Finger ${ }^{8}$, M. Finger Jr. ${ }^{8}$ 
Universidad San Francisco de Quito, Quito, Ecuador

E. Carrera Jarrin

Academy of Scientific Research and Technology of the Arab Republic of Egypt, Egyptian Network of High Energy Physics, Cairo, Egypt

A.A. Abdelalim ${ }^{9,10}$, A. Mohamed ${ }^{10}$, E. Salama ${ }^{11,12}$

National Institute of Chemical Physics and Biophysics, Tallinn, Estonia

S. Bhowmik, R.K. Dewanjee, M. Kadastik, L. Perrini, M. Raidal, C. Veelken

Department of Physics, University of Helsinki, Helsinki, Finland

P. Eerola, H. Kirschenmann, J. Pekkanen, M. Voutilainen

Helsinki Institute of Physics, Helsinki, Finland

J. Havukainen, J.K. Heikkilä, T. Järvinen, V. Karimäki, R. Kinnunen, T. Lampén,

K. Lassila-Perini, S. Laurila, S. Lehti, T. Lindén, P. Luukka, T. Mäenpää, H. Siikonen,

E. Tuominen, J. Tuominiemi

Lappeenranta University of Technology, Lappeenranta, Finland

T. Tuuva

IRFU, CEA, Université Paris-Saclay, Gif-sur-Yvette, France

M. Besancon, F. Couderc, M. Dejardin, D. Denegri, J.L. Faure, F. Ferri, S. Ganjour, S. Ghosh, A. Givernaud, P. Gras, G. Hamel de Monchenault, P. Jarry, C. Leloup, E. Locci, M. Machet, J. Malcles, G. Negro, J. Rander, A. Rosowsky, M.Ö. Sahin, M. Titov

Laboratoire Leprince-Ringuet, Ecole polytechnique, CNRS/IN2P3, Université Paris-Saclay, Palaiseau, France

A. Abdulsalam ${ }^{13}$, C. Amendola, I. Antropov, S. Baffioni, F. Beaudette, P. Busson,

L. Cadamuro, C. Charlot, R. Granier de Cassagnac, M. Jo, I. Kucher, S. Lisniak, A. Lobanov, J. Martin Blanco, M. Nguyen, C. Ochando, G. Ortona, P. Paganini, P. Pigard, R. Salerno, J.B. Sauvan, Y. Sirois, A.G. Stahl Leiton, Y. Yilmaz, A. Zabi, A. Zghiche

Université de Strasbourg, CNRS, IPHC UMR 7178, Strasbourg, France J.-L. Agram ${ }^{14}$, J. Andrea, D. Bloch, J.-M. Brom, E.C. Chabert, C. Collard, E. Conte ${ }^{14}$, X. Coubez, F. Drouhin ${ }^{14}$, J.-C. Fontaine ${ }^{14}$, D. Gelé, U. Goerlach, M. Jansová, P. Juillot, A.-C. Le Bihan, N. Tonon, P. Van Hove

Centre de Calcul de l'Institut National de Physique Nucleaire et de Physique des Particules, CNRS/IN2P3, Villeurbanne, France

S. Gadrat

Université de Lyon, Université Claude Bernard Lyon 1, CNRS-IN2P3, Institut de Physique Nucléaire de Lyon, Villeurbanne, France

S. Beauceron, C. Bernet, G. Boudoul, N. Chanon, R. Chierici, D. Contardo, P. Depasse, H. El Mamouni, J. Fay, L. Finco, S. Gascon, M. Gouzevitch, G. Grenier, B. Ille, F. Lagarde, I.B. Laktineh, H. Lattaud, M. Lethuillier, L. Mirabito, A.L. Pequegnot, S. Perries, A. Popov ${ }^{15}$, V. Sordini, M. Vander Donckt, S. Viret, S. Zhang 
Georgian Technical University, Tbilisi, Georgia

A. Khvedelidze ${ }^{8}$

Tbilisi State University, Tbilisi, Georgia

D. Lomidze

RWTH Aachen University, I. Physikalisches Institut, Aachen, Germany

C. Autermann, L. Feld, M.K. Kiesel, K. Klein, M. Lipinski, M. Preuten, M.P. Rauch, C. Schomakers, J. Schulz, M. Teroerde, B. Wittmer, V. Zhukov ${ }^{15}$

RWTH Aachen University, III. Physikalisches Institut A, Aachen, Germany

A. Albert, D. Duchardt, M. Endres, M. Erdmann, S. Erdweg, T. Esch, R. Fischer, A. Güth,

T. Hebbeker, C. Heidemann, K. Hoepfner, S. Knutzen, M. Merschmeyer, A. Meyer,

P. Millet, S. Mukherjee, T. Pook, M. Radziej, H. Reithler, M. Rieger, F. Scheuch,

D. Teyssier, S. Thüer

RWTH Aachen University, III. Physikalisches Institut B, Aachen, Germany

G. Flügge, B. Kargoll, T. Kress, A. Künsken, T. Müller, A. Nehrkorn, A. Nowack, C. Pistone, O. Pooth, A. Stahl ${ }^{16}$

\section{Deutsches Elektronen-Synchrotron, Hamburg, Germany}

M. Aldaya Martin, T. Arndt, C. Asawatangtrakuldee, K. Beernaert, O. Behnke, U. Behrens, A. Bermúdez Martínez, A.A. Bin Anuar, K. Borras ${ }^{17}$, V. Botta, A. Campbell, P. Connor, C. Contreras-Campana, F. Costanza, V. Danilov, A. De Wit, C. Diez Pardos, D. Domínguez Damiani, G. Eckerlin, D. Eckstein, T. Eichhorn, E. Eren, E. Gallo ${ }^{18}$, J. Garay Garcia, A. Geiser, J.M. Grados Luyando, A. Grohsjean, P. Gunnellini, M. Guthoff, A. Harb, J. Hauk, M. Hempel ${ }^{19}$, H. Jung, M. Kasemann, J. Keaveney, C. Kleinwort, J. Knolle, I. Korol, D. Krücker, W. Lange, A. Lelek, T. Lenz, K. Lipka, W. Lohmann ${ }^{19}$, R. Mankel, I.-A. Melzer-Pellmann, A.B. Meyer, M. Meyer, M. Missiroli, G. Mittag, J. Mnich, A. Mussgiller, D. Pitzl, A. Raspereza, M. Savitskyi, P. Saxena, R. Shevchenko, N. Stefaniuk, H. Tholen, G.P. Van Onsem, R. Walsh, Y. Wen, K. Wichmann, C. Wissing, O. Zenaiev

\section{University of Hamburg, Hamburg, Germany}

R. Aggleton, S. Bein, V. Blobel, M. Centis Vignali, T. Dreyer, E. Garutti, D. Gonzalez, J. Haller, A. Hinzmann, M. Hoffmann, A. Karavdina, G. Kasieczka, R. Klanner, R. Kogler, N. Kovalchuk, S. Kurz, J. Lange, D. Marconi, J. Multhaup, M. Niedziela, D. Nowatschin, T. Peiffer, A. Perieanu, A. Reimers, C. Scharf, P. Schleper, A. Schmidt, S. Schumann, J. Schwandt, J. Sonneveld, H. Stadie, G. Steinbrück, F.M. Stober, M. Stöver, D. Troendle, E. Usai, A. Vanhoefer, B. Vormwald

\section{Karlsruher Institut fuer Technology}

M. Akbiyik, C. Barth, M. Baselga, S. Baur, E. Butz, R. Caspart, T. Chwalek, F. Colombo, W. De Boer, A. Dierlamm, N. Faltermann, B. Freund, R. Friese, M. Giffels, M.A. Harrendorf, F. Hartmann ${ }^{16}$, S.M. Heindl, U. Husemann, F. Kassel ${ }^{16}$, S. Kudella, H. Mildner, M.U. Mozer, Th. Müller, M. Plagge, G. Quast, K. Rabbertz, M. Schröder, I. Shvetsov, 
G. Sieber, H.J. Simonis, R. Ulrich, S. Wayand, M. Weber, T. Weiler, S. Williamson, C. Wöhrmann, R. Wolf

Institute of Nuclear and Particle Physics (INPP), NCSR Demokritos, Aghia Paraskevi, Greece

G. Anagnostou, G. Daskalakis, T. Geralis, A. Kyriakis, D. Loukas, I. Topsis-Giotis

National and Kapodistrian University of Athens, Athens, Greece

G. Karathanasis, S. Kesisoglou, A. Panagiotou, N. Saoulidou, E. Tziaferi

National Technical University of Athens, Athens, Greece

K. Kousouris, I. Papakrivopoulos

University of Ioánnina, Ioánnina, Greece

I. Evangelou, C. Foudas, P. Gianneios, P. Katsoulis, P. Kokkas, S. Mallios, N. Manthos,

I. Papadopoulos, E. Paradas, J. Strologas, F.A. Triantis, D. Tsitsonis

MTA-ELTE Lendület CMS Particle and Nuclear Physics Group, Eötvös Loránd University, Budapest, Hungary

M. Csanad, N. Filipovic, G. Pasztor, O. Surányi, G.I. Veres ${ }^{20}$

Wigner Research Centre for Physics, Budapest, Hungary

G. Bencze, C. Hajdu, D. Horvath ${ }^{21}$, Á. Hunyadi, F. Sikler, T.Á. Vámi, V. Veszpremi,

G. Vesztergombi ${ }^{20}$

Institute of Nuclear Research ATOMKI, Debrecen, Hungary

N. Beni, S. Czellar, J. Karancsi22 ${ }^{2}$, A. Makovec, J. Molnar, Z. Szillasi

Institute of Physics, University of Debrecen, Debrecen, Hungary

M. Bartók ${ }^{20}$, P. Raics, Z.L. Trocsanyi, B. Ujvari

Indian Institute of Science (IISc), Bangalore, India

S. Choudhury, J.R. Komaragiri

National Institute of Science Education and Research, HBNI, Bhubaneswar, India

S. Bahinipati2 ${ }^{23}$, P. Mal, K. Mandal, A. Nayak ${ }^{24}$, D.K. Sahoo ${ }^{23}$, S.K. Swain

Panjab University, Chandigarh, India

S. Bansal, S.B. Beri, V. Bhatnagar, S. Chauhan, R. Chawla, N. Dhingra, R. Gupta, A. Kaur, M. Kaur, S. Kaur, R. Kumar, P. Kumari, M. Lohan, A. Mehta, S. Sharma, J.B. Singh, G. Walia

University of Delhi, Delhi, India

A. Bhardwaj, B.C. Choudhary, R.B. Garg, S. Keshri, A. Kumar, Ashok Kumar, S. Malhotra, M. Naimuddin, K. Ranjan, Aashaq Shah, R. Sharma

Saha Institute of Nuclear Physics, HBNI, Kolkata, India

R. Bhardwaj ${ }^{25}$, R. Bhattacharya, S. Bhattacharya, U. Bhawandeep ${ }^{25}$, D. Bhowmik, S. Dey, S. Dutt ${ }^{25}$, S. Dutta, S. Ghosh, N. Majumdar, K. Mondal, S. Mukhopadhyay, S. Nandan, 
A. Purohit, P.K. Rout, A. Roy, S. Roy Chowdhury, S. Sarkar, M. Sharan, B. Singh, S. Thakur ${ }^{25}$

\section{Indian Institute of Technology Madras, Madras, India}

P.K. Behera

\section{Bhabha Atomic Research Centre, Mumbai, India}

R. Chudasama, D. Dutta, V. Jha, V. Kumar, A.K. Mohanty ${ }^{16}$, P.K. Netrakanti, L.M. Pant, P. Shukla, A. Topkar

Tata Institute of Fundamental Research-A, Mumbai, India

T. Aziz, S. Dugad, B. Mahakud, S. Mitra, G.B. Mohanty, N. Sur, B. Sutar

Tata Institute of Fundamental Research-B, Mumbai, India

S. Banerjee, S. Bhattacharya, S. Chatterjee, P. Das, M. Guchait, Sa. Jain, S. Kumar, M. Maity ${ }^{26}$, G. Majumder, K. Mazumdar, N. Sahoo, T. Sarkar ${ }^{26}$, N. Wickramage ${ }^{27}$

Indian Institute of Science Education and Research (IISER), Pune, India

S. Chauhan, S. Dube, V. Hegde, A. Kapoor, K. Kothekar, S. Pandey, A. Rane, S. Sharma

Institute for Research in Fundamental Sciences (IPM), Tehran, Iran

S. Chenarani ${ }^{28}$, E. Eskandari Tadavani, S.M. Etesami ${ }^{28}$, M. Khakzad, M. Mohammadi Najafabadi, M. Naseri, S. Paktinat Mehdiabadi ${ }^{29}$, F. Rezaei Hosseinabadi, B. Safarzadeh ${ }^{30}$, M. Zeinali

\section{University College Dublin, Dublin, Ireland}

M. Felcini, M. Grunewald

INFN Sezione di Bari ${ }^{a}$, Università di Bari ${ }^{b}$, Politecnico di Bari ${ }^{c}$, Bari, Italy M. Abbrescia ${ }^{a, b}$, C. Calabria ${ }^{a, b}$ A. Colaleo ${ }^{a}$, D. Creanza ${ }^{a, c}$, L. Cristella ${ }^{a, b}$, N. De Filippis ${ }^{a, c}$, M. De Palma ${ }^{a, b}$, A. Di Florio ${ }^{a}, b$, F. Errico ${ }^{a, b}$, L. Fiore $^{a}$, A. Gelmi ${ }^{a, b}$,

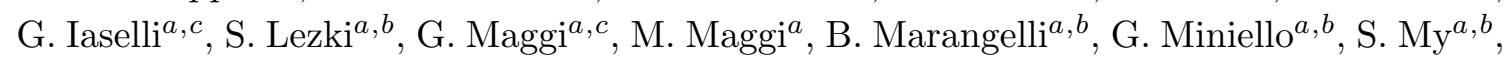
S. Nuzzo ${ }^{a, b}$, A. Pompilia ${ }^{a, b}$, G. Pugliese ${ }^{a, c}$, R. Radogna ${ }^{a}$, A. Ranieri ${ }^{a}$, G. Selvaggi ${ }^{a}, b$, A. Sharma ${ }^{a}$, L. Silvestris ${ }^{a}, 16$, R. Venditti ${ }^{a}$, P. Verwilligen ${ }^{a}$, G. Zito ${ }^{a}$

INFN Sezione di Bologna ${ }^{a}$, Università di Bologna ${ }^{b}$, Bologna, Italy

G. Abbiendi ${ }^{a}$, C. Battilana ${ }^{a, b}$, D. Bonacorsi ${ }^{a}, b$, L. Borgonovi $^{a, b}$, S. Braibant-Giacomelli ${ }^{a, b}$, R. Campanini ${ }^{a, b}$, P. Capiluppi ${ }^{a, b}$, A. Castro ${ }^{a, b}$, F.R. Cavallo ${ }^{a}$, S.S. Chhibra ${ }^{a, b}$, G. Codispoti ${ }^{a, b}$, M. Cuffiani ${ }^{a, b}$, G.M. Dallavalle ${ }^{a}$, F. Fabbri $^{a}$, A. Fanfani ${ }^{a, b}$, D. Fasanella $^{a, b}$, P. Giacomelli ${ }^{a}$, C. Grandi ${ }^{a}$, L. Guiducci ${ }^{a}, b$, S. Marcellini ${ }^{a}$, G. Masetti ${ }^{a}$, A. Montanari ${ }^{a}$, F.L. Navarria ${ }^{a, b}$, F. Odorici ${ }^{a}$, A. Perrotta ${ }^{a}$, A.M. Rossi ${ }^{a, b}$, T. Rovelli ${ }^{a, b}$, G.P. Siroli $^{a, b}$, N. Tosi ${ }^{a}$

INFN Sezione di Catania ${ }^{a}$, Università di Catania ${ }^{b}$, Catania, Italy

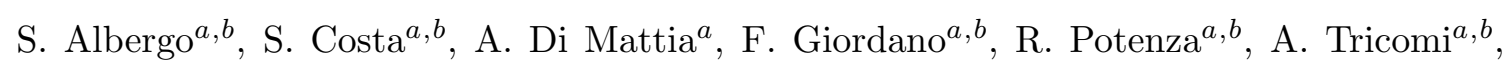
C. Tuve ${ }^{a, b}$ 
INFN Sezione di Firenze ${ }^{a}$, Università di Firenze ${ }^{b}$, Firenze, Italy

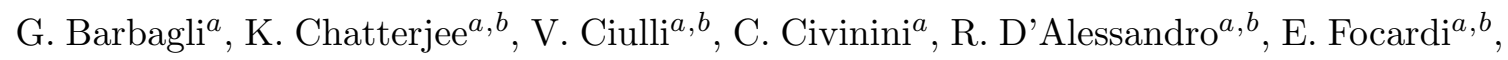

G. Latino, P. Lenzi ${ }^{a, b}$, M. Meschini ${ }^{a}$, S. Paoletti ${ }^{a}$, L. Russo $^{a, 31}$, G. Sguazzoni $^{a}$, D. Strom ${ }^{a}$,

L. Viliani ${ }^{a}$

INFN Laboratori Nazionali di Frascati, Frascati, Italy

L. Benussi, S. Bianco, F. Fabbri, D. Piccolo, F. Primavera ${ }^{16}$

INFN Sezione di Genova ${ }^{a}$, Università di Genova ${ }^{b}$, Genova, Italy

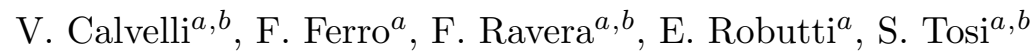

INFN Sezione di Milano-Bicocca ${ }^{a}$, Università di Milano-Bicocca ${ }^{b}$, Milano, Italy

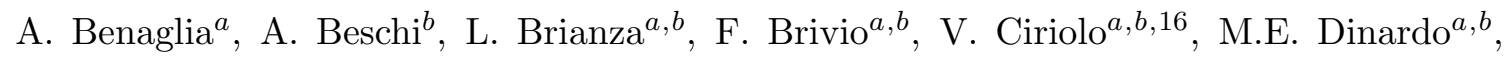
S. Fiorendi ${ }^{a, b}$, S. Gennai ${ }^{a}$, A. Ghezzi ${ }^{a, b}$, P. Govoni ${ }^{a, b}$, M. Malberti ${ }^{a, b}$, S. Malvezzi ${ }^{a}$, R.A. Manzoni ${ }^{a, b}$, D. Menasce ${ }^{a}$, L. Moroni $^{a}$, M. Paganoni ${ }^{a, b}$, K. Pauwels $^{a, b}$, D. Pedrini ${ }^{a}$, S. Pigazzini ${ }^{a, b, 32}$, S. Ragazzi ${ }^{a, b}$, T. Tabarelli de Fatis ${ }^{a, b}$

INFN Sezione di Napoli ${ }^{a}$, Università di Napoli 'Federico II' ${ }^{b}$, Napoli, Italy, Università della Basilicata ${ }^{c}$, Potenza, Italy, Università G. Marconi ${ }^{d}$, Roma, Italy

S. Buontempo ${ }^{a}$, N. Cavallo ${ }^{a, c}$, S. Di Guida ${ }^{a, d, 16}$, F. Fabozzi ${ }^{a, c}$, F. Fienga ${ }^{a, b}$, G. Galati ${ }^{a, b}$,

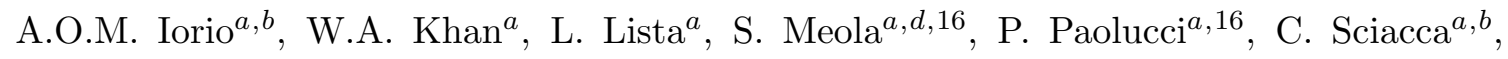
F. Thyssen ${ }^{a}$, E. Voevodina ${ }^{a, b}$

INFN Sezione di Padova ${ }^{a}$, Università di Padova ${ }^{b}$, Padova, Italy, Università di Trento ${ }^{c}$, Trento, Italy

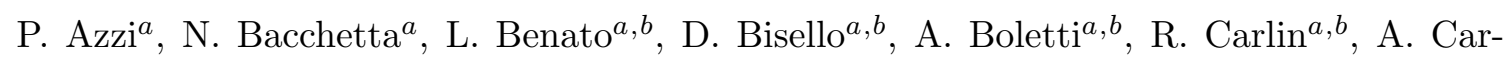
valho Antunes De Oliveira ${ }^{a, b}$, P. Checchia ${ }^{a}$, M. Dall'Osso ${ }^{a}, b$, P. De Castro Manzano ${ }^{a}$, T. Dorigo ${ }^{a}$, U. Dosselli ${ }^{a}$, U. Gasparini ${ }^{a, b}$, S. Lacaprara ${ }^{a}$, P. Lujan, M. Margoni ${ }^{a, b}$, A.T. Meneguzzo ${ }^{a, b}$, M. Michelotto ${ }^{a}$, N. Pozzobon ${ }^{a, b}$, P. Ronchese ${ }^{a, b}$, R. $\operatorname{Rossin}^{a, b}$, F. Simonetto ${ }^{a, b}$, A. Tiko, E. Torassa ${ }^{a}$, M. Zanetti ${ }^{a, b}$, P. Zotto ${ }^{a, b}$, G. Zumerle ${ }^{a, b}$

INFN Sezione di Pavia ${ }^{a}$, Università di Pavia ${ }^{b}$, Pavia, Italy

A. Braghieri ${ }^{a}$, A. Magnani ${ }^{a}$, P. Montagna ${ }^{a, b}$, S.P. Ratti ${ }^{a}, b$, V. $\operatorname{Re}^{a}$, M. Ressegotti $^{a, b}$, C. Riccardi ${ }^{a}, b$ P. Salvini ${ }^{a}$, I. Vai $^{a, b}$, P. Vitulo ${ }^{a, b}$

INFN Sezione di Perugia ${ }^{a}$, Università di Perugia ${ }^{b}$, Perugia, Italy

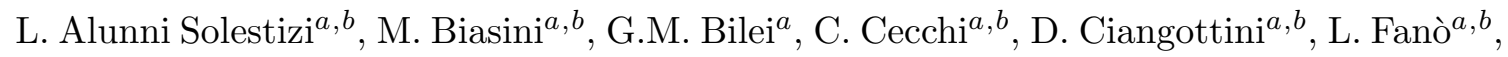
P. Lariccia ${ }^{a, b}$, R. Leonardi ${ }^{a, b}$, E. Manoni $^{a}$, G. Mantovani ${ }^{a, b}$, V. Mariani $^{a, b}$, M. Menichelli $^{a}$, A. $\operatorname{Rossi}^{a, b}$, A. Santocchia ${ }^{a, b}$, D. Spiga ${ }^{a}$

INFN Sezione di Pisa ${ }^{a}$, Università di Pisa ${ }^{b}$, Scuola Normale Superiore di Pisa ${ }^{c}$, Pisa, Italy

K. Androsov ${ }^{a}$, P. Azzurri ${ }^{a}, 16$, G. Bagliesi ${ }^{a}$, L. Bianchini ${ }^{a}$, T. Boccali ${ }^{a}$, L. Borrello,

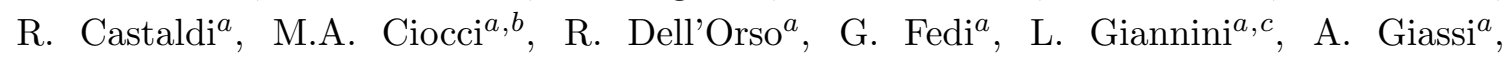
M.T. Grippo ${ }^{a, 31}$, F. Ligabue ${ }^{a, c}$, T. Lomtadze ${ }^{a}$, A. Malara, E. Manca ${ }^{a, c}$, G. Mandorli ${ }^{a, c}$, 


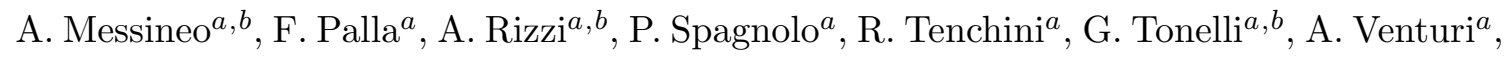
P.G. Verdini ${ }^{a}$

INFN Sezione di Roma ${ }^{a}$, Sapienza Università di Roma ${ }^{b}$, Rome, Italy

L. Barone ${ }^{a, b}$, F. Cavallari ${ }^{a}$, M. Cipriani ${ }^{a, b}$, N. Daci ${ }^{a}$, D. Del Re ${ }^{a, b}$, E. Di Marco ${ }^{a, b}$, M. Diemoz ${ }^{a}$, S. Gelli ${ }^{a, b}$, E. Longo ${ }^{a, b}$, B. Marzocchi ${ }^{a}, b$, P. Meridiani ${ }^{a}$, G. Organtini ${ }^{a, b}$, F. Pandolfi ${ }^{a}$, R. Paramatti ${ }^{a, b}$, F. Preiato ${ }^{a, b}$, S. Rahatlou $^{a, b}$, C. Rovelli $^{a}$, F. Santanastasio $^{a, b}$

INFN Sezione di Torino ${ }^{a}$, Università di Torino ${ }^{b}$, Torino, Italy, Università del Piemonte Orientale ${ }^{c}$, Novara, Italy

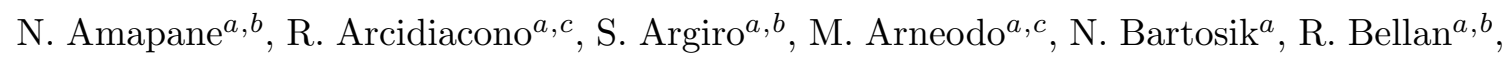

C. Biino ${ }^{a}$, N. Cartiglia ${ }^{a}$, R. Castello ${ }^{a, b}$, F. Cenna ${ }^{a, b}$, M. Costa ${ }^{a, b}$, R. Covarelli ${ }^{a}, b$,

A. $\operatorname{Degano}^{a, b}$, N. Demaria ${ }^{a}$, B. Kiani ${ }^{a, b}$, C. Mariotti ${ }^{a}$, S. Masellia ${ }^{a}$, E. Migliore ${ }^{a, b}$, V. Monaco ${ }^{a, b}$, E. Monteil $^{a, b}$, M. Monteno $^{a}$, M.M. Obertino ${ }^{a, b}$, L. Pacher $^{a, b}$, N. Pastrone $^{a}$, M. Pelliccioni ${ }^{a}$, G.L. Pinna Angioni ${ }^{a, b}$, A. Romero ${ }^{a, b}$, M. Ruspa ${ }^{a, c}$, R. Sacchi ${ }^{a, b}$, K. Shchelina ${ }^{a, b}$, V. Sola ${ }^{a}$, A. Solano ${ }^{a, b}$, A. Staiano ${ }^{a}$

INFN Sezione di Trieste ${ }^{a}$, Università di Trieste ${ }^{b}$, Trieste, Italy

S. Belforte ${ }^{a}$, M. Casarsa ${ }^{a}$, F. Cossutti ${ }^{a}$, G. Della Ricca ${ }^{a}, b$, A. Zanetti ${ }^{a}$

Kyungpook National University

D.H. Kim, G.N. Kim, M.S. Kim, J. Lee, S. Lee, S.W. Lee, C.S. Moon, Y.D. Oh, S. Sekmen, D.C. Son, Y.C. Yang

Chonnam National University, Institute for Universe and Elementary Particles, Kwangju, Korea

H. Kim, D.H. Moon, G. Oh

Hanyang University, Seoul, Korea

J.A. Brochero Cifuentes, J. Goh, T.J. Kim

Korea University, Seoul, Korea

S. Cho, S. Choi, Y. Go, D. Gyun, S. Ha, B. Hong, Y. Jo, Y. Kim, K. Lee, K.S. Lee, S. Lee, J. Lim, S.K. Park, Y. Roh

Seoul National University, Seoul, Korea

J. Almond, J. Kim, J.S. Kim, H. Lee, K. Lee, K. Nam, S.B. Oh, B.C. Radburn-Smith, S.h. Seo, U.K. Yang, H.D. Yoo, G.B. Yu

University of Seoul, Seoul, Korea

H. Kim, J.H. Kim, J.S.H. Lee, I.C. Park

Sungkyunkwan University, Suwon, Korea

Y. Choi, C. Hwang, J. Lee, I. Yu

Vilnius University, Vilnius, Lithuania

V. Dudenas, A. Juodagalvis, J. Vaitkus 
National Centre for Particle Physics, Universiti Malaya, Kuala Lumpur, Malaysia

I. Ahmed, Z.A. Ibrahim, M.A.B. Md $\mathrm{Ali}^{33}$, F. Mohamad Idris ${ }^{34}$, W.A.T. Wan Abdullah, M.N. Yusli, Z. Zolkapli

Centro de Investigacion y de Estudios Avanzados del IPN, Mexico City, Mexico H. Castilla-Valdez, E. De La Cruz-Burelo, M.C. Duran-Osuna, I. Heredia-De La Cruz ${ }^{35}$, R. Lopez-Fernandez, J. Mejia Guisao, R.I. Rabadan-Trejo, G. Ramirez-Sanchez, R ReyesAlmanza, A. Sanchez-Hernandez

Universidad Iberoamericana, Mexico City, Mexico

S. Carrillo Moreno, C. Oropeza Barrera, F. Vazquez Valencia

Benemerita Universidad Autonoma de Puebla, Puebla, Mexico

J. Eysermans, I. Pedraza, H.A. Salazar Ibarguen, C. Uribe Estrada

Universidad Autónoma de San Luis Potosí, San Luis Potosí, Mexico

A. Morelos Pineda

University of Auckland, Auckland, New Zealand

D. Krofcheck

University of Canterbury, Christchurch, New Zealand

S. Bheesette, P.H. Butler

National Centre for Physics, Quaid-I-Azam University, Islamabad, Pakistan

A. Ahmad, M. Ahmad, Q. Hassan, H.R. Hoorani, A. Saddique, M.A. Shah, M. Shoaib, M. Waqas

National Centre for Nuclear Research, Swierk, Poland

H. Bialkowska, M. Bluj, B. Boimska, T. Frueboes, M. Górski, M. Kazana, K. Nawrocki, M. Szleper, P. Traczyk, P. Zalewski

Institute of Experimental Physics, Faculty of Physics, University of Warsaw, Warsaw, Poland

K. Bunkowski, A. Byszuk ${ }^{36}$, K. Doroba, A. Kalinowski, M. Konecki, J. Krolikowski, M. Misiura, M. Olszewski, A. Pyskir, M. Walczak

Laboratório de Instrumentação e Física Experimental de Partículas, Lisboa, Portugal

P. Bargassa, C. Beirão Da Cruz E Silva, A. Di Francesco, P. Faccioli, B. Galinhas, M. Gallinaro, J. Hollar, N. Leonardo, L. Lloret Iglesias, M.V. Nemallapudi, J. Seixas, G. Strong, O. Toldaiev, D. Vadruccio, J. Varela

Joint Institute for Nuclear Research, Dubna, Russia

S. Afanasiev, V. Alexakhin, P. Bunin, M. Gavrilenko, A. Golunov, I. Golutvin, N. Gorbounov, V. Karjavin, A. Lanev, A. Malakhov, V. Matveev ${ }^{37,38}$, P. Moisenz, V. Palichik, V. Perelygin, M. Savina, S. Shmatov, V. Smirnov, N. Voytishin, A. Zarubin 
Petersburg Nuclear Physics Institute, Gatchina (St. Petersburg), Russia

Y. Ivanov, V. Kim ${ }^{39}$, E. Kuznetsova ${ }^{40}$, P. Levchenko, V. Murzin, V. Oreshkin, I. Smirnov,

D. Sosnov, V. Sulimov, L. Uvarov, S. Vavilov, A. Vorobyev

Institute for Nuclear Research, Moscow, Russia

Yu. Andreev, A. Dermenev, S. Gninenko, N. Golubev, A. Karneyeu, M. Kirsanov,

N. Krasnikov, A. Pashenkov, D. Tlisov, A. Toropin

Institute for Theoretical and Experimental Physics, Moscow, Russia

V. Epshteyn, V. Gavrilov, N. Lychkovskaya, V. Popov, I. Pozdnyakov, G. Safronov, A. Spiridonov, A. Stepennov, V. Stolin, M. Toms, E. Vlasov, A. Zhokin

Moscow Institute of Physics and Technology, Moscow, Russia

T. Aushev, A. Bylinkin ${ }^{38}$

National Research Nuclear University 'Moscow Engineering Physics Institute' (MEPhI), Moscow, Russia

R. Chistov ${ }^{41}$, M. Danilov ${ }^{41}$, P. Parygin, D. Philippov, S. Polikarpov, E. Tarkovskii

P.N. Lebedev Physical Institute, Moscow, Russia

V. Andreev, M. Azarkin ${ }^{38}$, I. Dremin ${ }^{38}$, M. Kirakosyan ${ }^{38}$, S.V. Rusakov, A. Terkulov

Skobeltsyn Institute of Nuclear Physics, Lomonosov Moscow State University, Moscow, Russia

A. Baskakov, A. Belyaev, E. Boos, V. Bunichev, M. Dubinin ${ }^{42}$, L. Dudko, A. Ershov,

V. Klyukhin, O. Kodolova, I. Lokhtin, I. Miagkov, S. Obraztsov, S. Petrushanko, V. Savrin,

A. Snigirev

Novosibirsk State University (NSU), Novosibirsk, Russia

V. Blinov ${ }^{43}$, D. Shtol ${ }^{43}$, Y. Skovpen ${ }^{43}$

State Research Center of Russian Federation, Institute for High Energy Physics of NRC "Kurchatov Institute", Protvino, Russia

I. Azhgirey, I. Bayshev, S. Bitioukov, D. Elumakhov, A. Godizov, V. Kachanov, A. Kalinin,

D. Konstantinov, P. Mandrik, V. Petrov, R. Ryutin, A. Sobol, S. Troshin, N. Tyurin,

A. Uzunian, A. Volkov

National Research Tomsk Polytechnic University, Tomsk, Russia

A. Babaev

University of Belgrade, Faculty of Physics and Vinca Institute of Nuclear Sciences, Belgrade, Serbia

P. Adzic ${ }^{44}$, P. Cirkovic, D. Devetak, M. Dordevic, J. Milosevic

Centro de Investigaciones Energéticas Medioambientales y Tecnológicas (CIEMAT), Madrid, Spain

J. Alcaraz Maestre, A. Álvarez Fernández, I. Bachiller, M. Barrio Luna, M. Cerrada, N. Colino, B. De La Cruz, A. Delgado Peris, C. Fernandez Bedoya, J.P. Fernández Ramos, J. Flix, M.C. Fouz, O. Gonzalez Lopez, S. Goy Lopez, J.M. Hernandez, M.I. Josa, D. Moran, 
A. Pérez-Calero Yzquierdo, J. Puerta Pelayo, I. Redondo, L. Romero, M.S. Soares, A. Triossi

\section{Universidad Autónoma de Madrid, Madrid, Spain}

C. Albajar, J.F. de Trocóniz

\section{Universidad de Oviedo, Oviedo, Spain}

J. Cuevas, C. Erice, J. Fernandez Menendez, S. Folgueras, I. Gonzalez Caballero, J.R. González Fernández, E. Palencia Cortezon, S. Sanchez Cruz, P. Vischia, J.M. Vizan Garcia

Instituto de Física de Cantabria (IFCA), CSIC-Universidad de Cantabria, Santander, Spain

I.J. Cabrillo, A. Calderon, B. Chazin Quero, J. Duarte Campderros, M. Fernandez, P.J. Fernández Manteca, A. García Alonso, J. Garcia-Ferrero, G. Gomez, A. Lopez Virto, J. Marco, C. Martinez Rivero, P. Martinez Ruiz del Arbol, F. Matorras, J. Piedra Gomez, C. Prieels, T. Rodrigo, A. Ruiz-Jimeno, L. Scodellaro, N. Trevisani, I. Vila, R. Vilar Cortabitarte

\section{CERN, European Organization for Nuclear Research, Geneva, Switzerland}

D. Abbaneo, B. Akgun, E. Auffray, P. Baillon, A.H. Ball, D. Barney, J. Bendavid, M. Bianco, A. Bocci, C. Botta, T. Camporesi, M. Cepeda, G. Cerminara, E. Chapon, Y. Chen, D. d'Enterria, A. Dabrowski, V. Daponte, A. David, M. De Gruttola, A. De Roeck, N. Deelen, M. Dobson, T. du Pree, M. Dünser, N. Dupont, A. Elliott-Peisert, P. Everaerts, F. Fallavollita ${ }^{45}$, G. Franzoni, J. Fulcher, W. Funk, D. Gigi, A. Gilbert, K. Gill, F. Glege, D. Gulhan, J. Hegeman, V. Innocente, A. Jafari, P. Janot, O. Karacheban ${ }^{19}$, J. Kieseler, V. Knünz, A. Kornmayer, M. Krammer ${ }^{1}$, C. Lange, P. Lecoq, C. Lourenço, M.T. Lucchini, L. Malgeri, M. Mannelli, A. Martelli, F. Meijers, J.A. Merlin, S. Mersi, E. Meschi, P. Milenovic ${ }^{46}$, F. Moortgat, M. Mulders, H. Neugebauer, J. Ngadiuba, S. Orfanelli, L. Orsini, F. Pantaleo ${ }^{16}$, L. Pape, E. Perez, M. Peruzzi, A. Petrilli, G. Petrucciani, A. Pfeiffer, M. Pierini, F.M. Pitters, D. Rabady, A. Racz, T. Reis, G. Rolandi ${ }^{47}$, M. Rovere, H. Sakulin, C. Schäfer, C. Schwick, M. Seidel, M. Selvaggi, A. Sharma, P. Silva, P. Sphicas ${ }^{48}$, A. Stakia, J. Steggemann, M. Stoye, M. Tosi, D. Treille, A. Tsirou, V. Veckalns ${ }^{49}$, M. Verweij, W.D. Zeuner

\section{Paul Scherrer Institut, Villigen, Switzerland}

W. Bertl ${ }^{\dagger}$, L. Caminada ${ }^{50}$, K. Deiters, W. Erdmann, R. Horisberger, Q. Ingram, H.C. Kaestli, D. Kotlinski, U. Langenegger, T. Rohe, S.A. Wiederkehr

ETH Zurich - Institute for Particle Physics and Astrophysics (IPA), Zurich, Switzerland

M. Backhaus, L. Bäni, P. Berger, B. Casal, N. Chernyavskaya, G. Dissertori, M. Dittmar, M. Donegà, C. Dorfer, C. Grab, C. Heidegger, D. Hits, J. Hoss, T. Klijnsma, W. Lustermann, M. Marionneau, M.T. Meinhard, D. Meister, F. Micheli, P. Musella, F. NessiTedaldi, J. Pata, F. Pauss, G. Perrin, L. Perrozzi, M. Quittnat, M. Reichmann, D. Ruini, 
D.A. Sanz Becerra, M. Schönenberger, L. Shchutska, V.R. Tavolaro, K. Theofilatos, M.L. Vesterbacka Olsson, R. Wallny, D.H. Zhu

Universität Zürich, Zurich, Switzerland

T.K. Aarrestad, C. Amsler ${ }^{51}$, D. Brzhechko, M.F. Canelli, A. De Cosa, R. Del Burgo, S. Donato, C. Galloni, T. Hreus, B. Kilminster, I. Neutelings, D. Pinna, G. Rauco, P. Robmann, D. Salerno, K. Schweiger, C. Seitz, Y. Takahashi, A. Zucchetta

National Central University, Chung-Li, Taiwan

V. Candelise, Y.H. Chang, K.y. Cheng, T.H. Doan, Sh. Jain, R. Khurana, C.M. Kuo,

W. Lin, A. Pozdnyakov, S.S. Yu

National Taiwan University (NTU), Taipei, Taiwan

P. Chang, Y. Chao, K.F. Chen, P.H. Chen, F. Fiori, W.-S. Hou, Y. Hsiung, Arun Kumar, Y.F. Liu, R.-S. Lu, E. Paganis, A. Psallidas, A. Steen, J.f. Tsai

Chulalongkorn University, Faculty of Science, Department of Physics, Bangkok, Thailand

B. Asavapibhop, K. Kovitanggoon, G. Singh, N. Srimanobhas

Çukurova University, Physics Department, Science and Art Faculty, Adana, Turkey

M.N. Bakirci ${ }^{52}$, A. Bat, F. Boran, S. Damarseckin, Z.S. Demiroglu, C. Dozen, E. Eskut, S. Girgis, G. Gokbulut, Y. Guler, I. Hos $^{53}$, E.E. Kangal ${ }^{54}$, O. Kara, U. Kiminsu, M. Oglakci, G. Onengut, K. Ozdemir ${ }^{55}$, S. Ozturk ${ }^{52}$, A. Polatoz, D. Sunar Cerci ${ }^{56}$, U.G. Tok, S. Turkcapar, I.S. Zorbakir, C. Zorbilmez

Middle East Technical University, Physics Department, Ankara, Turkey

G. Karapinar ${ }^{57}$, K. Ocalan ${ }^{58}$, M. Yalvac, M. Zeyrek

Bogazici University, Istanbul, Turkey

I.O. Atakisi, E. Gülmez, M. Kaya ${ }^{59}$, O. Kaya ${ }^{60}$, S. Tekten, E.A. Yetkin ${ }^{61}$

Istanbul Technical University, Istanbul, Turkey

M.N. Agaras, S. Atay, A. Cakir, K. Cankocak, Y. Komurcu

Institute for Scintillation Materials of National Academy of Science of Ukraine, Kharkov, Ukraine

B. Grynyov

National Scientific Center, Kharkov Institute of Physics and Technology, Kharkov, Ukraine

L. Levchuk

University of Bristol, Bristol, United Kingdom

F. Ball, L. Beck, J.J. Brooke, D. Burns, E. Clement, D. Cussans, O. Davignon, H. Flacher, J. Goldstein, G.P. Heath, H.F. Heath, L. Kreczko, D.M. Newbold ${ }^{62}$, S. Paramesvaran, T. Sakuma, S. Seif El Nasr-storey, D. Smith, V.J. Smith 


\section{Rutherford Appleton Laboratory, Didcot, United Kingdom}

K.W. Bell, A. Belyaev ${ }^{63}$, C. Brew, R.M. Brown, D. Cieri, D.J.A. Cockerill, J.A. Coughlan, K. Harder, S. Harper, J. Linacre, E. Olaiya, D. Petyt, C.H. Shepherd-Themistocleous, A. Thea, I.R. Tomalin, T. Williams, W.J. Womersley

\section{Imperial College, London, United Kingdom}

G. Auzinger, R. Bainbridge, P. Bloch, J. Borg, S. Breeze, O. Buchmuller, A. Bundock, S. Casasso, D. Colling, L. Corpe, P. Dauncey, G. Davies, M. Della Negra, R. Di Maria, A. Elwood, Y. Haddad, G. Hall, G. Iles, T. James, M. Komm, R. Lane, C. Laner, L. Lyons, A.-M. Magnan, S. Malik, L. Mastrolorenzo, T. Matsushita, J. $\mathrm{Nash}^{64}$, A. Nikitenko ${ }^{7}$, V. Palladino, M. Pesaresi, A. Richards, A. Rose, E. Scott, C. Seez, A. Shtipliyski, T. Strebler, S. Summers, A. Tapper, K. Uchida, M. Vazquez Acosta ${ }^{65}$, T. Virdee ${ }^{16}$, N. Wardle, D. Winterbottom, J. Wright, S.C. Zenz

Brunel University, Uxbridge, United Kingdom

J.E. Cole, P.R. Hobson, A. Khan, P. Kyberd, A. Morton, I.D. Reid, L. Teodorescu, S. Zahid Baylor University, Waco, U.S.A.

A. Borzou, K. Call, J. Dittmann, K. Hatakeyama, H. Liu, N. Pastika, C. Smith

Catholic University of America, Washington DC, U.S.A.

R. Bartek, A. Dominguez

The University of Alabama, Tuscaloosa, U.S.A.

A. Buccilli, S.I. Cooper, C. Henderson, P. Rumerio, C. West

Boston University, Boston, U.S.A.

D. Arcaro, A. Avetisyan, T. Bose, D. Gastler, D. Rankin, C. Richardson, J. Rohlf, L. Sulak, D. Zou

\section{Brown University, Providence, U.S.A.}

G. Benelli, D. Cutts, M. Hadley, J. Hakala, U. Heintz, J.M. Hogan ${ }^{66}$, K.H.M. Kwok, E. Laird, G. Landsberg, J. Lee, Z. Mao, M. Narain, J. Pazzini, S. Piperov, S. Sagir, R. Syarif, D. Yu

University of California, Davis, Davis, U.S.A.

R. Band, C. Brainerd, R. Breedon, D. Burns, M. Calderon De La Barca Sanchez, M. Chertok, J. Conway, R. Conway, P.T. Cox, R. Erbacher, C. Flores, G. Funk, W. Ko, R. Lander, C. Mclean, M. Mulhearn, D. Pellett, J. Pilot, S. Shalhout, M. Shi, J. Smith, D. Stolp, D. Taylor, K. Tos, M. Tripathi, Z. Wang, F. Zhang

University of California, Los Angeles, U.S.A.

M. Bachtis, C. Bravo, R. Cousins, A. Dasgupta, A. Florent, J. Hauser, M. Ignatenko, N. Mccoll, S. Regnard, D. Saltzberg, C. Schnaible, V. Valuev

University of California, Riverside, Riverside, U.S.A.

E. Bouvier, K. Burt, R. Clare, J. Ellison, J.W. Gary, S.M.A. Ghiasi Shirazi, G. Hanson, G. Karapostoli, E. Kennedy, F. Lacroix, O.R. Long, M. Olmedo Negrete, M.I. Paneva, W. Si, L. Wang, H. Wei, S. Wimpenny, B.R. Yates 
University of California, San Diego, La Jolla, U.S.A.

J.G. Branson, S. Cittolin, M. Derdzinski, R. Gerosa, D. Gilbert, B. Hashemi, A. Holzner, D. Klein, G. Kole, V. Krutelyov, J. Letts, M. Masciovecchio, D. Olivito, S. Padhi, M. Pieri, M. Sani, V. Sharma, S. Simon, M. Tadel, A. Vartak, S. Wasserbaech ${ }^{67}$, J. Wood, F. Würthwein, A. Yagil, G. Zevi Della Porta

University of California, Santa Barbara - Department of Physics, Santa Barbara, U.S.A.

N. Amin, R. Bhandari, J. Bradmiller-Feld, C. Campagnari, M. Citron, A. Dishaw, V. Dutta, M. Franco Sevilla, L. Gouskos, R. Heller, J. Incandela, A. Ovcharova, H. Qu, J. Richman, D. Stuart, I. Suarez, J. Yoo

California Institute of Technology, Pasadena, U.S.A.

D. Anderson, A. Bornheim, J. Bunn, J.M. Lawhorn, H.B. Newman, T.Q. Nguyen, C. Pena, M. Spiropulu, J.R. Vlimant, R. Wilkinson, S. Xie, Z. Zhang, R.Y. Zhu

Carnegie Mellon University, Pittsburgh, U.S.A.

M.B. Andrews, T. Ferguson, T. Mudholkar, M. Paulini, J. Russ, M. Sun, H. Vogel, I. Vorobiev, M. Weinberg

University of Colorado Boulder, Boulder, U.S.A.

J.P. Cumalat, W.T. Ford, F. Jensen, A. Johnson, M. Krohn, S. Leontsinis, E. MacDonald, T. Mulholland, K. Stenson, K.A. Ulmer, S.R. Wagner

Cornell University, Ithaca, U.S.A.

J. Alexander, J. Chaves, Y. Cheng, J. Chu, A. Datta, K. Mcdermott, N. Mirman, J.R. Patterson, D. Quach, A. Rinkevicius, A. Ryd, L. Skinnari, L. Soffi, S.M. Tan, Z. Tao, J. Thom, J. Tucker, P. Wittich, M. Zientek

Fermi National Accelerator Laboratory, Batavia, U.S.A.

S. Abdullin, M. Albrow, M. Alyari, G. Apollinari, A. Apresyan, A. Apyan, S. Banerjee, L.A.T. Bauerdick, A. Beretvas, J. Berryhill, P.C. Bhat, G. Bolla ${ }^{\dagger}$, K. Burkett, J.N. Butler, A. Canepa, G.B. Cerati, H.W.K. Cheung, F. Chlebana, M. Cremonesi, J. Duarte, V.D. Elvira, J. Freeman, Z. Gecse, E. Gottschalk, L. Gray, D. Green, S. Grünendahl, O. Gutsche, J. Hanlon, R.M. Harris, S. Hasegawa, J. Hirschauer, Z. Hu, B. Jayatilaka, S. Jindariani, M. Johnson, U. Joshi, B. Klima, M.J. Kortelainen, B. Kreis, S. Lammel, D. Lincoln, R. Lipton, M. Liu, T. Liu, R. Lopes De Sá, J. Lykken, K. Maeshima, N. Magini, J.M. Marraffino, D. Mason, P. McBride, P. Merkel, S. Mrenna, S. Nahn, V. O'Dell, K. Pedro, O. Prokofyev, G. Rakness, L. Ristori, A. Savoy-Navarro ${ }^{68}$, B. Schneider, E. Sexton-Kennedy, A. Soha, W.J. Spalding, L. Spiegel, S. Stoynev, J. Strait, N. Strobbe, L. Taylor, S. Tkaczyk, N.V. Tran, L. Uplegger, E.W. Vaandering, C. Vernieri, M. Verzocchi, R. Vidal, M. Wang, H.A. Weber, A. Whitbeck, W. Wu

University of Florida, Gainesville, U.S.A.

D. Acosta, P. Avery, P. Bortignon, D. Bourilkov, A. Brinkerhoff, A. Carnes, M. Carver, D. Curry, R.D. Field, I.K. Furic, S.V. Gleyzer, D. Guerrero, B.M. Joshi, J. Konigsberg, 
A. Korytov, K. Kotov, P. Ma, K. Matchev, H. Mei, G. Mitselmakher, K. Shi, D. Sperka, N. Terentyev, L. Thomas, J. Wang, S. Wang, J. Yelton

Florida International University, Miami, U.S.A.

Y.R. Joshi, S. Linn, P. Markowitz, J.L. Rodriguez

Florida State University, Tallahassee, U.S.A.

A. Ackert, T. Adams, A. Askew, S. Hagopian, V. Hagopian, K.F. Johnson, T. Kolberg, G. Martinez, T. Perry, H. Prosper, A. Saha, A. Santra, V. Sharma, R. Yohay

Florida Institute of Technology, Melbourne, U.S.A.

M.M. Baarmand, V. Bhopatkar, S. Colafranceschi, M. Hohlmann, D. Noonan, T. Roy, F. Yumiceva

University of Illinois at Chicago (UIC), Chicago, U.S.A.

M.R. Adams, L. Apanasevich, D. Berry, R.R. Betts, R. Cavanaugh, X. Chen, S. Dittmer, O. Evdokimov, C.E. Gerber, D.A. Hangal, D.J. Hofman, K. Jung, J. Kamin, I.D. Sandoval Gonzalez, M.B. Tonjes, N. Varelas, H. Wang, Z. Wu, J. Zhang

The University of Iowa, Iowa City, U.S.A.

B. Bilki ${ }^{69}$, W. Clarida, K. Dilsiz ${ }^{70}$, S. Durgut, R.P. Gandrajula, M. Haytmyradov, V. Khristenko, J.-P. Merlo, H. Mermerkaya ${ }^{71}$, A. Mestvirishvili, A. Moeller, J. Nachtman, H. Ogul ${ }^{72}$, Y. Onel, F. Ozok ${ }^{73}$, A. Penzo, C. Snyder, E. Tiras, J. Wetzel, K. Yi

Johns Hopkins University, Baltimore, U.S.A.

B. Blumenfeld, A. Cocoros, N. Eminizer, D. Fehling, L. Feng, A.V. Gritsan, W.T. Hung, P. Maksimovic, J. Roskes, U. Sarica, M. Swartz, M. Xiao, C. You

The University of Kansas, Lawrence, U.S.A.

A. Al-bataineh, P. Baringer, A. Bean, S. Boren, J. Bowen, J. Castle, S. Khalil, A. Kropivnitskaya, D. Majumder, W. Mcbrayer, M. Murray, C. Rogan, C. Royon, S. Sanders, E. Schmitz, J.D. Tapia Takaki, Q. Wang

Kansas State University, Manhattan, U.S.A.

A. Ivanov, K. Kaadze, Y. Maravin, A. Modak, A. Mohammadi, L.K. Saini, N. Skhirtladze

Lawrence Livermore National Laboratory, Livermore, U.S.A.

F. Rebassoo, D. Wright

University of Maryland, College Park, U.S.A.

A. Baden, O. Baron, A. Belloni, S.C. Eno, Y. Feng, C. Ferraioli, N.J. Hadley, S. Jabeen, G.Y. Jeng, R.G. Kellogg, J. Kunkle, A.C. Mignerey, F. Ricci-Tam, Y.H. Shin, A. Skuja, S.C. Tonwar

\section{Massachusetts Institute of Technology, Cambridge, U.S.A.}

D. Abercrombie, B. Allen, V. Azzolini, R. Barbieri, A. Baty, G. Bauer, R. Bi, S. Brandt, W. Busza, I.A. Cali, M. D’Alfonso, Z. Demiragli, G. Gomez Ceballos, M. Goncharov, P. Harris, D. Hsu, M. Hu, Y. Iiyama, G.M. Innocenti, M. Klute, D. Kovalskyi, Y.-J. Lee, A. Levin, P.D. Luckey, B. Maier, A.C. Marini, C. Mcginn, C. Mironov, S. Narayanan, 
X. Niu, C. Paus, C. Roland, G. Roland, G.S.F. Stephans, K. Sumorok, K. Tatar, D. Velicanu, J. Wang, T.W. Wang, B. Wyslouch, S. Zhaozhong

University of Minnesota, Minneapolis, U.S.A.

A.C. Benvenuti, R.M. Chatterjee, A. Evans, P. Hansen, S. Kalafut, Y. Kubota, Z. Lesko, J. Mans, S. Nourbakhsh, N. Ruckstuhl, R. Rusack, J. Turkewitz, M.A. Wadud

University of Mississippi, Oxford, U.S.A.

J.G. Acosta, S. Oliveros

University of Nebraska-Lincoln, Lincoln, U.S.A.

E. Avdeeva, K. Bloom, D.R. Claes, C. Fangmeier, F. Golf, R. Gonzalez Suarez, R. Kamalieddin, I. Kravchenko, J. Monroy, J.E. Siado, G.R. Snow, B. Stieger

State University of New York at Buffalo, Buffalo, U.S.A.

C. Harrington, I. Iashvili, A. Kharchilava, D. Nguyen, A. Parker, S. Rappoccio, B. Roozbahani

Northeastern University, Boston, U.S.A.

G. Alverson, E. Barberis, C. Freer, A. Hortiangtham, A. Massironi, D.M. Morse, T. Orimoto, R. Teixeira De Lima, T. Wamorkar, B. Wang, A. Wisecarver, D. Wood

Northwestern University, Evanston, U.S.A.

S. Bhattacharya, O. Charaf, K.A. Hahn, N. Mucia, N. Odell, M.H. Schmitt, K. Sung, M. Trovato, M. Velasco

University of Notre Dame, Notre Dame, U.S.A.

R. Bucci, N. Dev, M. Hildreth, K. Hurtado Anampa, C. Jessop, D.J. Karmgard, N. Kellams, K. Lannon, W. Li, N. Loukas, N. Marinelli, F. Meng, C. Mueller, Y. Musienko ${ }^{37}$, M. Planer, A. Reinsvold, R. Ruchti, P. Siddireddy, G. Smith, S. Taroni, M. Wayne, A. Wightman, M. Wolf, A. Woodard

The Ohio State University, Columbus, U.S.A.

J. Alimena, L. Antonelli, B. Bylsma, L.S. Durkin, S. Flowers, B. Francis, A. Hart, C. Hill, W. Ji, T.Y. Ling, W. Luo, B.L. Winer, H.W. Wulsin

Princeton University, Princeton, U.S.A.

S. Cooperstein, O. Driga, P. Elmer, J. Hardenbrook, P. Hebda, S. Higginbotham, A. Kalogeropoulos, D. Lange, J. Luo, D. Marlow, K. Mei, I. Ojalvo, J. Olsen, C. Palmer, P. Piroué, J. Salfeld-Nebgen, D. Stickland, C. Tully

University of Puerto Rico, Mayaguez, U.S.A.

S. Malik, S. Norberg

Purdue University, West Lafayette, U.S.A.

A. Barker, V.E. Barnes, S. Das, L. Gutay, M. Jones, A.W. Jung, A. Khatiwada, D.H. Miller, N. Neumeister, C.C. Peng, H. Qiu, J.F. Schulte, J. Sun, F. Wang, R. Xiao, W. Xie

Purdue University Northwest, Hammond, U.S.A.

T. Cheng, J. Dolen, N. Parashar 
Rice University, Houston, U.S.A.

Z. Chen, K.M. Ecklund, S. Freed, F.J.M. Geurts, M. Guilbaud, M. Kilpatrick, W. Li, B. Michlin, B.P. Padley, J. Roberts, J. Rorie, W. Shi, Z. Tu, J. Zabel, A. Zhang

University of Rochester, Rochester, U.S.A.

A. Bodek, P. de Barbaro, R. Demina, Y.t. Duh, T. Ferbel, M. Galanti, A. Garcia-Bellido, J. Han, O. Hindrichs, A. Khukhunaishvili, K.H. Lo, P. Tan, M. Verzetti

The Rockefeller University, New York, U.S.A.

R. Ciesielski, K. Goulianos, C. Mesropian

Rutgers, The State University of New Jersey, Piscataway, U.S.A.

A. Agapitos, J.P. Chou, Y. Gershtein, T.A. Gómez Espinosa, E. Halkiadakis, M. Heindl,

E. Hughes, S. Kaplan, R. Kunnawalkam Elayavalli, S. Kyriacou, A. Lath, R. Montalvo,

K. Nash, M. Osherson, H. Saka, S. Salur, S. Schnetzer, D. Sheffield, S. Somalwar, R. Stone,

S. Thomas, P. Thomassen, M. Walker

University of Tennessee, Knoxville, U.S.A.

A.G. Delannoy, J. Heideman, G. Riley, K. Rose, S. Spanier, K. Thapa

Texas A\&M University, College Station, U.S.A.

O. Bouhali ${ }^{74}$, A. Castaneda Hernandez ${ }^{74}$, A. Celik, M. Dalchenko, M. De Mattia, A. Delgado, S. Dildick, R. Eusebi, J. Gilmore, T. Huang, T. Kamon ${ }^{75}$, R. Mueller, Y. Pakhotin, R. Patel, A. Perloff, L. Perniè, D. Rathjens, A. Safonov, A. Tatarinov

Texas Tech University, Lubbock, U.S.A.

N. Akchurin, J. Damgov, F. De Guio, P.R. Dudero, J. Faulkner, E. Gurpinar, S. Kunori, K. Lamichhane, S.W. Lee, T. Mengke, S. Muthumuni, T. Peltola, S. Undleeb, I. Volobouev, Z. Wang

Vanderbilt University, Nashville, U.S.A.

S. Greene, A. Gurrola, R. Janjam, W. Johns, C. Maguire, A. Melo, H. Ni, K. Padeken, J.D. Ruiz Alvarez, P. Sheldon, S. Tuo, J. Velkovska, Q. Xu

University of Virginia, Charlottesville, U.S.A.

M.W. Arenton, P. Barria, B. Cox, R. Hirosky, M. Joyce, A. Ledovskoy, H. Li, C. Neu, T. Sinthuprasith, Y. Wang, E. Wolfe, F. Xia

Wayne State University, Detroit, U.S.A.

R. Harr, P.E. Karchin, N. Poudyal, J. Sturdy, P. Thapa, S. Zaleski

University of Wisconsin - Madison, Madison, WI, U.S.A.

M. Brodski, J. Buchanan, C. Caillol, D. Carlsmith, S. Dasu, L. Dodd, S. Duric, B. Gomber, M. Grothe, M. Herndon, A. Hervé, U. Hussain, P. Klabbers, A. Lanaro, A. Levine, K. Long, R. Loveless, V. Rekovic, T. Ruggles, A. Savin, N. Smith, W.H. Smith, N. Woods 
$\dagger$ : Deceased

1: Also at Vienna University of Technology, Vienna, Austria

2: Also at IRFU, CEA, Université Paris-Saclay, Gif-sur-Yvette, France

3: Also at Universidade Estadual de Campinas, Campinas, Brazil

4: Also at Federal University of Rio Grande do Sul, Porto Alegre, Brazil

5: Also at Universidade Federal de Pelotas, Pelotas, Brazil

6: Also at Université Libre de Bruxelles, Bruxelles, Belgium

7: Also at Institute for Theoretical and Experimental Physics, Moscow, Russia

8: Also at Joint Institute for Nuclear Research, Dubna, Russia

9: Also at Helwan University, Cairo, Egypt

10: Now at Zewail City of Science and Technology, Zewail, Egypt

11: Also at British University in Egypt, Cairo, Egypt

12: Now at Ain Shams University, Cairo, Egypt

13: Also at Department of Physics, King Abdulaziz University, Jeddah, Saudi Arabia

14: Also at Université de Haute Alsace, Mulhouse, France

15: Also at Skobeltsyn Institute of Nuclear Physics, Lomonosov Moscow State University, Moscow, Russia

16: Also at CERN, European Organization for Nuclear Research, Geneva, Switzerland

17: Also at RWTH Aachen University, III. Physikalisches Institut A, Aachen, Germany

18: Also at University of Hamburg, Hamburg, Germany

19: Also at Brandenburg University of Technology, Cottbus, Germany

20: Also at MTA-ELTE Lendület CMS Particle and Nuclear Physics Group, Eötvös Loránd University, Budapest, Hungary

21: Also at Institute of Nuclear Research ATOMKI, Debrecen, Hungary

22: Also at Institute of Physics, University of Debrecen, Debrecen, Hungary

23: Also at Indian Institute of Technology Bhubaneswar, Bhubaneswar, India

24: Also at Institute of Physics, Bhubaneswar, India

25: Also at Shoolini University, Solan, India

26: Also at University of Visva-Bharati, Santiniketan, India

27: Also at University of Ruhuna, Matara, Sri Lanka

28: Also at Isfahan University of Technology, Isfahan, Iran

29: Also at Yazd University, Yazd, Iran

30: Also at Plasma Physics Research Center, Science and Research Branch, Islamic Azad University, Tehran, Iran

31: Also at Università degli Studi di Siena, Siena, Italy

32: Also at INFN Sezione di Milano-Bicocca ${ }^{a}$, Università di Milano-Bicocca ${ }^{b}$, Milano, Italy

33: Also at International Islamic University of Malaysia, Kuala Lumpur, Malaysia

34: Also at Malaysian Nuclear Agency, MOSTI, Kajang, Malaysia

35: Also at Consejo Nacional de Ciencia y Tecnología, Mexico city, Mexico

36: Also at Warsaw University of Technology, Institute of Electronic Systems, Warsaw, Poland

37: Also at Institute for Nuclear Research, Moscow, Russia

38: Now at National Research Nuclear University 'Moscow Engineering Physics Institute' (MEPhI), Moscow, Russia

39: Also at St. Petersburg State Polytechnical University, St. Petersburg, Russia

40: Also at University of Florida, Gainesville, U.S.A.

41: Also at P.N. Lebedev Physical Institute, Moscow, Russia

42: Also at California Institute of Technology, Pasadena, U.S.A.

43: Also at Budker Institute of Nuclear Physics, Novosibirsk, Russia 
44: Also at Faculty of Physics, University of Belgrade, Belgrade, Serbia

45: Also at INFN Sezione di Pavia ${ }^{a}$, Università di Pavia ${ }^{b}$, Pavia, Italy

46: Also at University of Belgrade, Faculty of Physics and Vinca Institute of Nuclear Sciences, Belgrade, Serbia

47: Also at Scuola Normale e Sezione dell'INFN, Pisa, Italy

48: Also at National and Kapodistrian University of Athens, Athens, Greece

49: Also at Riga Technical University, Riga, Latvia

50: Also at Universität Zürich, Zurich, Switzerland

51: Also at Stefan Meyer Institute for Subatomic Physics (SMI), Vienna, Austria

52: Also at Gaziosmanpasa University, Tokat, Turkey

53: Also at Istanbul Aydin University, Istanbul, Turkey

54: Also at Mersin University, Mersin, Turkey

55: Also at Piri Reis University, Istanbul, Turkey

56: Also at Adiyaman University, Adiyaman, Turkey

57: Also at Izmir Institute of Technology, Izmir, Turkey

58: Also at Necmettin Erbakan University, Konya, Turkey

59: Also at Marmara University, Istanbul, Turkey

60: Also at Kafkas University, Kars, Turkey

61: Also at Istanbul Bilgi University, Istanbul, Turkey

62: Also at Rutherford Appleton Laboratory, Didcot, United Kingdom

63: Also at School of Physics and Astronomy, University of Southampton, Southampton, United Kingdom

64: Also at Monash University, Faculty of Science, Clayton, Australia

65: Also at Instituto de Astrofísica de Canarias, La Laguna, Spain

66: Also at Bethel University, St. Paul, U.S.A.

67: Also at Utah Valley University, Orem, U.S.A.

68: Also at Purdue University, West Lafayette, U.S.A.

69: Also at Beykent University, Istanbul, Turkey

70: Also at Bingol University, Bingol, Turkey

71: Also at Erzincan University, Erzincan, Turkey

72: Also at Sinop University, Sinop, Turkey

73: Also at Mimar Sinan University, Istanbul, Istanbul, Turkey

74: Also at Texas A\&M University at Qatar, Doha, Qatar

75: Also at Kyungpook National University, Daegu, Korea 